\title{
Averaging and spectral properties for the 2D advection-diffusion equation in the semi-classical limit for vanishing diffusivity
}

\author{
J. Vukadinovic ${ }^{\mathrm{a}, \mathrm{b}, \mathrm{c}}$, E. Dedits ${ }^{\mathrm{a}}$, A. C. Poje ${ }^{\mathrm{a}, \mathrm{c}}$, T. Schäfer ${ }^{\mathrm{a}, \mathrm{c}, *}$ \\ ${ }^{a}$ Physics Program at the CUNY Graduate Center \\ 365 5th Ave, New York, NY 10016 \\ ${ }^{b}$ Mathematics Program at the CUNY Graduate Center \\ 365 5th Ave, New York, NY 10016 \\ ${ }^{c}$ Department of Mathematics, College of Staten Island \\ 2800 Victory Blvd, Staten Island, NY 10314
}

\begin{abstract}
We consider the two-dimensional advection-diffusion equation on a bounded domain subject to Dirichlet or von Neumann boundary conditions involving a Liouville integrable Hamiltonian. Transformation to action-angle coordinates permits averaging in time and angle, resulting in an equation that allows for separation of variables. The Fourier transform in the angle coordinate transforms the equation into an effective diffusive equation and a countable family of non-self-adjoint Schrödinger equations. For the corresponding Liouville-Sturm problem, we apply complex-plane WKB methods to study the spectrum in the semi-classical limit for vanishing diffusivity. The spectral limit graph is found to consist of analytic curves (branches) related to Stokes graphs forming a tree-structure. Eigenvalues in the neighborhood of branches emanating from the imaginary axis are subject to various sublinear power laws with respect to diffusivity, leading to convection-enhanced rates of dissipation of the corresponding modes. The solution of ADE converges in the limit of vanishing diffusivity to the solution of the effective diffusion equation on convective time scales that are sublinear with respect to the diffusive time scales.
\end{abstract}

Keywords: advection-diffusion equation, averaging, convection-enhanced

${ }^{*}$ Corresponding author. Phone: 718982 3616. Fax: 7819823631

Email address: tobias@math.csi.cuny.edu (T. Schäfer)

Preprint submitted to Physica D

July 22, 2015

(C) 2015. This manuscript version is made available under the Elsevier user license http://www.elsevier.com/open-access/userlicense/1.0/ 
mixing, WKB method

\section{Introduction}

We study the Cauchy problem for the advection-diffusion equation (ADE) in the 'small-diffusion' formulation,

$$
c_{t}+(\mathbf{u} \cdot \nabla) c=\varepsilon \Delta c, \quad c(t=0)=\stackrel{\circ}{c} .
$$

We consider Dirichlet or von Neumann conditions on bounded domains in two spatial variables. The unknown $c(t, x, y)$ is a scalar function of spatial variables $(x, y)$ and time $t$, while $\mathbf{u}(t, x, y)$ is a given time-independent or time-periodic vector-field (flow). The constant $\varepsilon>0$ is a given, scaled diffusivity. We restrict our attention to divergence-free flows only, $\nabla \cdot \mathbf{u}(t, \cdot)=0$. More specifically, we assume the existence of a time-independent or timeperiodic stream-function (Hamiltonian), $\Psi(t, x, y)$, such that $\mathbf{u}(t, x, y)=$ $\nabla \times \Psi(t, x, y)$, with $\nabla \times=\left(\partial_{y},-\partial_{x}\right)$ denoting the two-dimensional curl.

The equation constitutes an important paradigm for a wide range of physical, chemical, and biological processes that are characterized both by transport induced by a fluid flow as well as small diffusion. Examples include homogenization in fluid mixtures, pollutant dispersion in the ocean or atmosphere, temporal evolution of biological systems in flowing media, energy transport in flowing media, etc. A significant volume of physical and mathematical literature has been devoted to the study of the ADE in various settings: on unbounded domains, compact manifolds, and bounded domains together with appropriate boundary conditions. The rather intricate interplay between diffusion and advection in the semi-classical limit for vanishing diffusivity, $\varepsilon \rightarrow 0$, remains an important area of active scientific inquiry. The convection-driven enhancement of the rate of dissipation, relaxation, or mixing is of particular interest. Depending on the type of the boundary value problem, various methods such as homogenization, probabilistic, variational and 'PDE' methods have been employed. In order to put our approach into perspective and historical context, we give a brief review of the relevant literature.

On unbounded domains and periodic media, the convection-driven enhancement of dissipation was addressed within the framework of the homogenization theory $[12,16,17]$. It was shown that the long-time asymptotic behavior of solutions is governed by an effective diffusion equation, 
$c_{t}=A^{\varepsilon}: \nabla \nabla c$, where the constant matrix $A^{\varepsilon}$ is the so-called effective diffusivity tensor. The effective diffusion in a given direction $\mathbf{e}$ is then given by $\mathbf{e}^{T} A^{\varepsilon} \mathbf{e}$, and various scaling regimes $\varepsilon^{\alpha}$ with $\alpha \leq 1$ as $\varepsilon \rightarrow 0$ have been identified. In this context, scaling regimes $\alpha<1$, and in particular $\alpha=1 / 2$ are referred to as convection-enhanced diffusion.

On a bounded domain $D$ together with Dirichlet boundary conditions, the mixing properties of solutions of the advection-diffusion equation are closely related to the structure of the spectrum of the non-self-adjoint advectiondiffusion operator $\mathcal{L}_{\varepsilon}=-\varepsilon \Delta+\mathbf{u} \cdot \nabla$. In the autonomous case and under some mild regularity conditions on $\mathbf{u}$, the advection-diffusion operator possesses a pure point spectrum consisting of isolated eigenvalues that have positive real part $[1,2]$. Of particular interest is the eigenvalue with the least positive real part, $\lambda_{0}^{\varepsilon}$, referred to as the principal eigenvalue. It determines the slowest time scale of dissipation in the sense that for generic initial data $\stackrel{\circ}{c}$,

$$
t^{-1} \log \left\|c^{(\varepsilon)}(t, \cdot)\right\|_{L^{2}(D)} \rightarrow-\lambda_{0}^{\varepsilon} \quad \text { as } \quad t \rightarrow \infty .
$$

Convection-enhanced dissipation is characterized by the sublinear dependence of $\lambda_{0}^{\varepsilon}$ on $\varepsilon$. Employing variational and PDE methods, Berestycki et al. [4] identified the sharp criterion that $\varepsilon^{-1} \lambda_{0}^{\varepsilon}$ is unbounded as $\varepsilon \rightarrow 0$ if and only if the only first integral $w$ of $\mathbf{u}(\mathbf{u} \cdot \nabla w=0)$ in the space $H_{0}^{1}(D)$ is trivial. This, in turn, is equivalent to the 'short-term' dissipation enhancement in the sense that the dissipation takes place on diffusive time scales $T_{D}^{(\varepsilon)} \sim \varepsilon^{-1}$, i.e.,

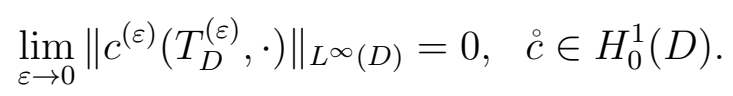

On compact manifolds or bounded domains together with von Neumann boundary conditions, the situation is slightly different in that the principal eigenvalue is always zero, and the long-term behavior is characterized by the relaxation to the average $\langle\dot{c}\rangle$. In this context, Constantin et al. [5] characterized dissipation enhancing flows $\mathbf{u}$ (flows for which $\lim _{\varepsilon \rightarrow 0} \| c^{(\varepsilon)}\left(T_{D}^{(\varepsilon)}\right)-$ $\left.\left\langle c{ }^{\circ}\right\rangle \|_{L^{\infty}(D)}=0\right)$, by the condition that the only eigenfunctions of the advection operator $\mathbf{u} \cdot \nabla$ in $H^{1}(D)$ are constant. On unbounded domains, Zlatoš characterized dissipation enhancing flows $\mathbf{u}$ by the condition that the only eigenfunctions of the advection operator $\mathbf{u} \cdot \nabla$ are the first integrals of $\mathbf{u}$ (see [35]).

There are many important examples of flows that are not dissipation enhancing, but for which convection still plays an important role in the mixing 
properties. These examples are addressed by the theory developed by Freidlin, Wentzell at al. [19, 20, 21, 22]. The authors use probabilistic methods to study ADE on a closed domain together with Dirichlet boundary conditions by viewing it as a random perturbation of a Hamiltonian system. The theory applies to the situation, in which the stream-function $\Psi$ satisfies certain non-degeneracy and growth conditions and the stream-lines are Jordan curves. In particular, these assumptions imply the existence of nontrivial first integrals in $H_{0}^{1}(D)$, hence the flow does not enhance dissipation in the sense of Berestycki et al. The main result of the theory is the convergence of the solutions of ADE on diffusive time scales to solutions of an effective diffusion equation on the Reeb graph of the Hamiltonian, which is obtained by collapsing each stream-line to a point. However, the theory falls somewhat short of explaining the exact role of convection in mixing, and the results are not sharp. It appears that the convergence of solutions of the advection-diffusion equation to solutions of the effective diffusive equation occurs on much shorter sublinear time scales than the diffusive time scales predicted by the theory of Freidlin and Wentzell. This observation has been repeatedly made in the physics literature [23, 24, 26, 25], but lacks a rigorous justification.

In this paper, we employ spectral theoretical methods to a special but instructive case from the Freidlin-Wentzell theory and prove convergence of solutions of ADE to those of the effective diffusion equation on 'convective' timescales $T_{C}^{(\varepsilon)} \sim \varepsilon^{-\eta}$, with $\eta \in(0,1)$. Our case is special in that the Hamiltonian is assumed to be completely integrable in the sense of Liouville, thus allowing transformation to action-angle coordinates $(x, y) \rightarrow$ $(J, \theta)$. The Hamiltonian can then be expressed as a function of the action variable, $H=h(J)$, and the advection operator assumes the simple form $\mathbf{u} \cdot \nabla=\omega(J) \partial_{\theta}$, where $\omega=d h / d J$. In the classical mechanics literature, the function $\omega$ is often referred to as the frequency (function), as it represents the angular frequency of the periodic motion associated with the Hamiltonian (see [34]). It may be obtained without finding a complete solution for the motion, once $h$ is determined as a function of the action variable $J$. The domain $D$ is typically an annular region $\left\{j^{-}<J<j^{+}\right\}$. Our approach consists of transforming the ADE to action-angle coordinates and averaging the coefficients of the Laplacian in time and angle coordinates. The advantage of the averaged equation is that it allows for separation of variables. The Fourier transform in the angle coordinate transforms it into a 1D effective diffusion equation and a countable family of 1D Schrödinger equations 
with an important caveat that they are non-self-adjoint, since the potential is imaginary-valued function involving the frequency function. For the corresponding non-self-adjoint Sturm-Liouville problems, we employ complexplane WKB techniques to study the complicated geometric structure of the limit spectral graph for vanishing diffusivity, and to characterize the various power scaling laws.

Numerical simulations have long suggested that the spectrum of the Dirichlet problem for the 1D Schrödinger operators with an imaginary potential converges in the semi-classical limit to a graph consisting of analytic curves exhibiting an intricate tree-structure. Upon fixing a potential within the Schrödinger operator, then in the semi-classical limit an eigenvalue branch emanates from each endpoint of the range of the potential, denoted $j^{ \pm}$, as well as from each extremal value of the potential. As the imaginary part of the points along the eigenvalue branches decreases, once two distinct eigenvalue branches collide in a node, they are replaced by another eigenvalue branch (see Fig. 1). This persists until there is only one eigenvalue branch remaining. In the context of the Orr-Sommerfeld problem, this observation has recently been confirmed analytically by Shkalikov et al. $[29,30,31]$, first for linear, and then for any analytic monotonic or parabolic potential. Here, we adapt their approach to the problem at hand, and, in addition, explicitly determine the sublinear power laws along each eigenvalue branch. These scaling laws directly determine the convective timescales of dissipation of dominant modes. It turns out that they are determined by the power of the leading term in the Taylor expansions of the frequency function about each endpoint $j^{ \pm}$and each critical point at which the frequency function has an extremum. In particular, if the power of the leading term in the Taylor expansion of the frequency function about a certain point $j$ is is $n$, then the power scaling law for the eigenvalue branch emanating from the value $\omega(j)$ is given by $\varepsilon^{\frac{n}{n+2}}$.

Let us point out that the exact structure of the limit spectral graph is well understood only for monotonic and parabolic analytic potentials, and it becomes significantly less tractable for a higher number of critical points. That is the reason why we focus on these simpler but representative cases. Most of our analysis is carried out for the monotonic frequency functions. In addition to monotonicity, we assume that there exists a domain $G \subset \mathbb{C}$ and a semi-strip $\Pi \subset \mathbb{C}$, such that $\omega: G \rightarrow \Pi$ is a univalent surjection such that $\frac{d}{d x}(\Re \omega) \geq 0$ on $G$. This, however, is not a very restrictive condition as long as $\omega$ is monotonic and has an analytic extension, and it is satisfied by a great 
number of examples.

The results are applicable to important examples of unidirectional axisymmetric radial flows in annular regions or disks, including physically realizable Couette flow and the MHD driven annular micromixers leading to Poiseuille profiles. In both cases, advection-enhanced diffusion has been previously studied and observed numerically [23, 24, 26, 25]. For the Couette profile, the frequency is a monotone function, hence there are only two convective eigenvalue branches emanating from each endpoint of the range of the frequency function. The Taylor expansion about the endpoints has a nontrivial linear term resulting in the power scaling law $\varepsilon^{1 / 3}$. For the Poiseuille profile, the frequency possesses a quadratic extremum resulting in the existence of a branch along which the eigenvalues obey the power scaling law $\varepsilon^{1 / 2}$. This is in addition to two branches emanating from endpoints, along which the eigenvalues obey the power law $\varepsilon^{1 / 3}$.

Once the geometric structure of the limit spectral graph and the scaling laws of the eigenvalues along different branches are established, we express the semigroup for the solution of the non-self-adjoint Schrödinger equation via a spectral expansion. However, while the non-orthogonal set of eigenfunctions is complete, the associated spectral expansions need not converge in $L^{2}$. We make use of the so-called Abel-Lidskii summation formula to infer dissipation rates for the $L^{2}$-norm of the solutions from the eigenvalue scaling laws. For the advection-diffusion equation on the other hand, the phase space is a direct sum of a 'diffusive' phase space and a 'convective' phase space, $H_{0}^{1}(D)=H_{D} \oplus H_{C}$, and the solutions can be written as

$$
c^{(\varepsilon)}(t)=c_{D}^{(\varepsilon)}(t)+c_{C}^{(\varepsilon)}(t)
$$

where $c_{D}^{(\varepsilon)}$ is a solution of the effective diffusion equation that dissipates on timescales that are superlinear to the diffusive ones, and $c_{C}^{(\varepsilon)}$ is the convective component that dissipates on shorter convective timescales $T_{C}^{(\varepsilon)} \sim \varepsilon^{-\eta}$ with $\eta \in(0,1)$, i.e.,

$$
\lim _{\varepsilon \rightarrow 0}\left\|c_{C}^{(\varepsilon)}\left(T_{C}^{(\varepsilon)}\right)\right\|_{L^{\infty}(D)}=0 .
$$

Strictly speaking, the dissipation rates of the solutions for generic initial data are not convection enhanced, as the diffusive component of the solution dissipates over longer than diffusive timescales. However, if we think of 'mixing' as dissipation of different modes at different rates (sublinear or superlinear), we can speak of convection-enhanced mixing rather than dissipation. 
We also address the non-autonomous case when the stream function is time-dependent and time-periodic. More specifically, we treat the case when for each time $t$, the stream function $\Psi(t)$ is completely integrable in the sense of of Liouville, and the corresponding transformation to action-angle coordinates $(x, y) \rightarrow(J, \theta)$ is time-independent. An additional transformation to a 'co-moving' reference frame allows for time-angle averaging, and the resulting averaged equation is up to a small diffusive correction an autonomous advection-diffusion equation. Hence, at least in this specific case, the mixing properties are qualitatively not very different for the non-autonomous advection equation than for the autonomous case described above.

\section{Averaging in action-angle coordinates and the averaged spectral problem}

\subsection{Autonomous case}

\subsubsection{Action-angle coordinates and angle-averaging}

Let a domain $D \subset \mathbb{R}^{2}$ be topologically equivalent to an annulus bounded by two Jordan curves $\mathcal{C}^{-}$and $\mathcal{C}^{+}$. We first consider the autonomous advectiondiffusion equation (1) with $\mathbf{u}(x, y)=\nabla \times H(x, y)$ for some time-independent Hamiltonian $H \in C^{2}(\bar{D})$, such that $H(x, y)=h^{-}$for $(x, y) \in \mathcal{C}^{-}$and $H(x, y)=h^{+}$for $(x, y) \in \mathcal{C}^{+}$. Further, we assume that the corresponding Hamiltonian system is completely integrable in the sense of Liouville. It is a well known result from classical mechanics (we refer the reader to [34]

for details) that the system then allows for a canonical transformation to action-angle coordinates,

$$
(x, y) \rightarrow(J, \theta),
$$

which in particular satisfy the following conditions: (a) the Hamiltonian can be expressed as a function of the action coordinate, $H=h(J)$; (b) $\oint d \theta=2 \pi$, with the integral taken over a connected component of a level set of $H$; (c) the variables $(J, \theta)$ are canonical coordinates. Introducing the frequency function $\omega=d h / d J$, the phase flow with Hamiltonian $H$ satisfies the equations

$$
\frac{d J}{d t}=0, \quad \frac{d \theta}{d t}=\omega(J)
$$

Note that there exist values $j^{-}$and $j^{+}$(w.l.o.g., $j^{-}<j^{+}$) such that $\mathcal{C}^{ \pm}=$ $\left\{(x, y): J(x, y)=j^{ \pm}\right\}$. We consider the Dirichlet boundary conditions $\left.c\right|_{\partial D}=$ 0 or the von Neuman boundary conditions $d c /\left.d J\right|_{\partial D}=0$. It is obvious 
that for any $g \in C^{1}\left(j^{-}, j^{+}\right)$such that $g\left(j^{-}\right)=g\left(j^{+}\right)=0$, the function $w(x, y)=g(J)$ is a first integral of $\mathbf{u}$ belonging to $H_{0}^{1}(D)$, and therefore the flow $\mathbf{u}$ is not dissipation enhancing in the sense of $(2)$.

Let us remark here as well that the case when $D$ is bounded and simply connected with $\partial D=\left\{(x, y): J(x, y)=j_{2}\right\}$ for some $j_{2}$ can be treated similarly. There exists $j^{-}$(w.l.o.g., $j^{-}<j^{+}$), so that $\left\{(x, y): J(x, y)=j^{-}\right\}$ consists of a point at which $\theta(x, y)$ is undefined. We then study the problem on the punctured disk $\left\{(x, y): j^{-}<J(x, y)<j^{+}\right\}$. The fact that the coefficients of the diffusion operator in action-angle coordinates possess a singularity at the punctured point imposes an asymptotic boundary condition at $j^{-}$, while at $j^{+}$we have the Dirichlet or von Neumann conditions.

The advection-diffusion equation (1) in action-angle coordinates can be written as

$$
c_{t}+\omega(J) c_{\theta}=\varepsilon \Delta_{(J, \theta)} c,
$$

where $\Delta_{(J, \theta)}=\left|\nabla_{(x, y)} J\right|^{2} \partial_{J J}+\left|\nabla_{(x, y)} \theta\right|^{2} \partial_{\theta \theta}+\left(\Delta_{(x, y)} J\right) \partial_{J}+\left(\Delta_{(x, y)} \theta\right) \partial_{\theta}$. We express the coefficients for the Laplacian in terms of action-angle coordinates, $a_{11}(J, \theta)=\left|\nabla_{(x, y)} J\right|^{2}, a_{22}(J, \theta)=\left|\nabla_{(x, y)} \theta\right|^{2}, b_{1}(J, \theta)=\Delta_{(x, y)} J$ and $b_{2}(J, \theta)=$ $\Delta_{(x, y)} \theta$, and introduce the notation

$$
\begin{gathered}
A(J, \theta): \nabla \nabla=a_{11}(J, \theta) \partial_{J J}+a_{22}(J, \theta) \partial_{\theta \theta}, \\
\mathbf{b}(J, \theta) \cdot \nabla=b_{1}(J, \theta) \partial_{J}+b_{2}(J, \theta) \partial_{\theta}, \\
\Delta_{(J, \theta)}=A(J, \theta): \nabla \nabla+\mathbf{b}(J, \theta) \cdot \nabla .
\end{gathered}
$$

For the averages along the streamlines, we use the notation $\langle c\rangle(J)=\frac{1}{2 \pi} \int_{0}^{2 \pi} c(J, \theta) d \theta$. Taking the averages in (4), we obtain the equation

$$
\langle c\rangle_{t}=\varepsilon \Delta_{J}\langle c\rangle
$$

and scalar multiplication in $L^{2}\left(j^{-}, j^{+}\right)$by $\langle c\rangle$ yields

$$
\frac{1}{2} \frac{d}{d t}\|\langle c\rangle\|_{L^{2}\left(j^{-}, j^{+}\right)}+\varepsilon\left\|\nabla_{J}\langle c\rangle\right\|_{L^{2}\left(j^{-}, j^{+}\right)}=0 .
$$

In particular, if $\left\langle{ }_{c}^{\circ}\right\rangle \equiv 0$, then $\langle c(t)\rangle \equiv 0, t \geq 0$. Note that the coefficient functions $a_{11}, a_{22}, b_{1}$ and $b_{2}$ are periodic in the angle coordinate $\theta$ with period $2 \pi$. Introducing the following notation for the averages

$$
\bar{a}_{i j}(J)=\left\langle a_{i j}\right\rangle(J)=\frac{1}{2 \pi} \int_{0}^{2 \pi} a_{i j}(J, \theta) d \theta
$$


and

$$
\bar{b}_{i}(J)=\left\langle b_{i}\right\rangle(J)=\frac{1}{2 \pi} \int_{0}^{2 \pi} b_{i}(J, \theta) d \theta,
$$

we write ADE with angle-averaged coefficients,

$$
v_{t}+\omega(J) v_{\theta}=\varepsilon(\langle A\rangle(J): \nabla \nabla+\langle\mathbf{b}\rangle(J) \cdot \nabla) v .
$$

Equation (4) can be viewed as a small zero-mean perturbation of equation (7). Let us denote

$$
\mathcal{B}(J, \theta)=(A(J, \theta)-\langle A\rangle(J)): \nabla \nabla+(\mathbf{b}(J, \theta)-\langle\mathbf{b}\rangle(J)) \cdot \nabla .
$$

It can be easily verified that if $c=v+\varepsilon \mathfrak{u}$ is a solution of (4) and $v$ a solution of (7), then $\mathfrak{u}$ satisfies

$$
\mathfrak{u}_{t}+\omega(J) \mathfrak{u}_{\theta}=\varepsilon(A(J, \theta): \nabla \nabla+\mathbf{b}(J, \theta) \cdot \nabla) \mathfrak{u}+\mathcal{B}(J, \theta) v .
$$

\subsubsection{Effective diffusion equation}

Rescaling equation (5) yields the so-called effective diffusion equation,

$$
\bar{v}_{t}=\left\langle a_{11}\right\rangle(J) \bar{v}_{J J}+\left\langle b_{1}\right\rangle(J) \bar{v}_{J} .
$$

Using probabilistic methods, it was proven (see [19, 20, 21, 22, 27]) that under the assumption that $d \omega / d J$ has a finite number of zeros, the solutions of (4) converge to the solutions of (9) in the limit $\varepsilon \rightarrow 0$. More precisely, the limit $\bar{c}(t, J):=\lim _{\varepsilon \rightarrow 0} c^{(\varepsilon)}\left(\varepsilon^{-1} t, J, \theta\right)$ exists, it does not depend on the angle coordinate $\theta$, and it solves the effective diffusion equation (9). Furthermore,

$$
\lim _{\varepsilon \rightarrow 0}\left\|c^{(\varepsilon)}\left(\varepsilon^{-1} t, \cdot\right)-\bar{v}(t, \cdot)\right\|_{\infty}=0
$$

In other words, on 'diffusive' time scales $T_{D}^{(\varepsilon)} \sim \varepsilon^{-1}$, the solutions of the advection-diffusion equation converge to the solutions of the effective diffusion equation. The purpose of this paper is to improve this result by showing that this convergence occurs on shorter 'convective' time scales $T_{C}^{(\varepsilon)} \sim \varepsilon^{-\eta}$ for some $\eta \in(0,1)$ by studying the semi-classical limit of the spectral boundary value problem. 


\subsubsection{Spectral problem for the averaged equation}

We introduce the advection-diffusion operator in action-angle coordinates,

$$
\mathcal{L}_{\varepsilon}=-\varepsilon \Delta_{(J, \theta)}+\omega(J) \partial_{\theta},
$$

and write the corresponding spectral boundary value problem

$$
\mathcal{L}_{\varepsilon} \phi=\lambda \phi
$$

Similarly, we introduce the advection-diffusion operator for equation (7),

$$
\left\langle\mathcal{L}_{\varepsilon}\right\rangle=-\varepsilon(\langle A\rangle(J): \nabla \nabla+\langle\mathbf{b}\rangle(J) \cdot \nabla)+\omega(J) \partial_{\theta} .
$$

The corresponding spectral boundary value problem then reads

$$
\left\langle\mathcal{L}_{\varepsilon}\right\rangle \phi=\lambda \phi .
$$

The (formal) adjoint of the operator $\left\langle\mathcal{L}_{\varepsilon}\right\rangle$ is given by

$$
\left\langle\mathcal{L}_{\varepsilon}\right\rangle^{*}=-\varepsilon(\langle A\rangle(J): \nabla \nabla+\langle\mathbf{b}\rangle(J) \cdot \nabla)-\omega(J) \partial_{\theta},
$$

and the corresponding spectral problem reads

$$
\left\langle\mathcal{L}_{\varepsilon}\right\rangle^{*} \hat{\phi}=\bar{\lambda} \hat{\phi}
$$

Regarding the spectrum for non-self-adjoint problems (11) and (12) we refer the reader to the following theorem due to Agmon (see[1, 2]).

Theorem 2.1. Let us assume the following:

1. $\Omega \subset \mathbb{R}^{d}$ is of class $C^{3,1}$;

2. $\mathcal{L}(x, D)=\sum_{|\alpha| \leq 2} a_{\alpha}(x) D^{\alpha}$ is uniformly strongly elliptic in $\Omega$, with $a_{\alpha}$, real-valued if $|\alpha|=2$ and complex-valued otherwise and such that $a_{\alpha} \in$ $C^{|\alpha|, 1}(\bar{\Omega})$ for $|\alpha| \geq 1$ and $a_{\alpha} \in L^{\infty}(\Omega)$ otherwise;

3. $B(x, D)=\sum_{|\alpha| \leq 1} b_{\alpha}(x) D^{\alpha}$, with $b$ real-valued if $|\alpha|=1$ and complexvalued otherwise, while $b_{\alpha} \in C^{|\alpha|+1,1}(\partial \Omega)$ for $|\alpha| \geq 0$;

4. $\partial \Omega$ is non-characteristic to $B$ at each of its points.

Then the elliptic boundary problem $\mathcal{L} u=\lambda u$ in $\Omega$ and $B u=0$ on $L^{2}(\partial \Omega)$ is regular. Let $D\left(A_{p}\right)=\left\{u \in W^{2, p}(\Omega): B u=0\right\}$ and $A_{p} u=\mathcal{L} u, u \in$ $D\left(A_{p}\right)$. Then $A_{p}$ has non-empty resolvent set, compact resolvent, and hence a discrete spectrum. Moreover, the eigenvalues and eigenvectors of $A_{p}$ are the same for all $p$. The spectrum consists solely of eigenvalues of finite algebraic multiplicity which form a denumerably infinite subset of $\mathbb{C}$ having no finite points of accumulation. Moreover, the eigenfunctions form a complete set in $L^{2}(\Omega)$, i.e., their span is dense in $L^{2}(\Omega)$. 
Problem (12) has the advantage that we can seek the eigenfunctions through the ansatz

$$
\phi_{m, n}(J, \theta)=e^{i m \theta} g_{m, n}(J),
$$

where $m$ and $n$ are integers. For a fixed 'quantum number' $m \in \mathbb{Z}$, we introduce the following non-self-adjoint Schrödinger operator,

$$
\left\langle\mathcal{L}_{m, \varepsilon}\right\rangle g=-\varepsilon\left(\left\langle a_{11}\right\rangle(J) g^{\prime \prime}+\left\langle b_{1}\right\rangle(J) g^{\prime}-m^{2}\left\langle a_{22}\right\rangle(J) g\right)+i m \omega(J) g,
$$

and its (formal) adjoint

$$
\left\langle\mathcal{L}_{m, \varepsilon}\right\rangle^{*} g=-\varepsilon\left(\left\langle a_{11}\right\rangle(J) g^{\prime \prime}+\left\langle b_{1}\right\rangle(J) g^{\prime}-m^{2}\left\langle a_{22}\right\rangle(J) g\right)-i m \omega(J) g .
$$

The functions $g_{m, n}(J)$ satisfy the spectral boundary value problem

$$
\left\langle\mathcal{L}_{m, \varepsilon}\right\rangle g_{m, n}=\lambda_{m, n} g_{m, n},
$$

together with the Dirichlet boundary condition $g_{m, n}\left(j_{1}\right)=g_{m, n}\left(j_{2}\right)=0$ (or von Neumann boundary conditions $\left.g_{m, n}^{\prime}\left(j_{1}\right)=g_{m, n}^{\prime}\left(j_{2}\right)=0\right)$. For $m=0$, equation (13) is the self-adjoint spectral problem associated with the effective diffusion equation,

$$
-\varepsilon\left(\left\langle a_{11}\right\rangle(J) g_{0, n}^{\prime \prime}+\left\langle b_{1}\right\rangle(J) g_{0, n}^{\prime}\right)=\lambda_{0, n} g_{0, n} .
$$

Clearly, the eigenvalues of this equation scale linearly with $\varepsilon$.

On the other hand, when $m \neq 0,(13)$ is a non-self-adjoint convectiondominated problem which is much more difficult to analyze. This will be the focus of the next section. However, it is easy to establish that the spectrum consists of eigenvalues of multiplicity one which form a denumerably infinite subset $\left\{\lambda_{m, n}\right\}_{n=1}^{\infty}$ of $\mathbb{C}$ having no finite points of accumulation. Moreover, the eigenfunctions $g_{m, n}, n \in \mathbb{N}$ form a complete set in $L^{2}\left(j^{-}, j^{+}\right)$, i.e., their span is dense in $L^{2}\left(j^{-}, j^{+}\right)$.

We shall refer to the eigenvalue/eigenfunction pairs $\left(\lambda_{m, n}, \phi_{m, n}\right)$ as 'diffusive' when $m=0$ and 'convective' for $m \neq 0$. Taking the closures in $H^{1}(D)$, we denote

$$
H_{m}=\overline{\operatorname{span}\left\{\phi_{m, n}: n \in \mathbb{N}\right\}}
$$

Obviously,

$$
H_{0}^{1}(D)=\oplus_{m \in \mathbb{Z}} H_{m}
$$


We also denote $H_{C}=H_{0}$, and $H_{D}=\oplus_{m \in \mathbb{Z}, m \neq 0} H_{m}$. The phase space of initial data for (7) can now be written as a direct sum,

$$
H_{0}^{1}(D)=H_{D} \oplus H_{C}
$$

Note, however, that because of (6), the phase space of initial data for (4) can be written in the same way.

For $\stackrel{v}{\in} H_{0}^{1}(D)$ let

$$
\stackrel{\circ}{v}_{m}(J):=\frac{1}{2 \pi} \int_{0}^{2 \pi} \stackrel{v}{v}(J, \theta) e^{-i m \theta} d \theta,
$$

and

$$
P_{m} \stackrel{\circ}{v}=\stackrel{\circ}{v}_{m}(J) e^{i m \theta} .
$$

Then $\stackrel{\circ}{v}_{m} \in L^{2}\left(j^{-}, j^{+}\right), m \in \mathbb{Z}$, and the following expansion holds in $L^{2}(D)$ :

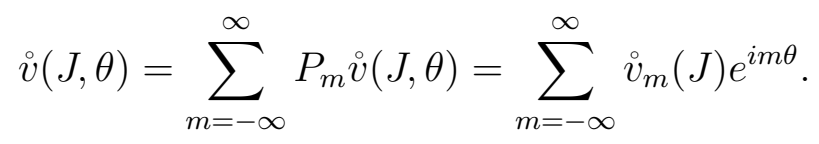

Moreover,

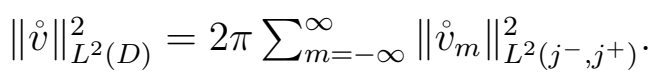

Denote by $v_{m}(t)$ the solution to

$$
\frac{\partial v_{m}}{\partial t}+i m \omega v_{m}=\varepsilon\left(\left\langle a_{11}\right\rangle \frac{\partial^{2} v_{m}}{\partial J^{2}}+\left\langle b_{1}\right\rangle \frac{\partial v_{m}}{\partial J}-m^{2}\left\langle a_{22}\right\rangle v_{m}\right), v_{m}(0)=\stackrel{\circ}{v}_{m} .
$$

The solution to equation (7) is then given by

$$
v(t, J, \theta)=\sum_{m=-\infty}^{\infty} v_{m}(t, J) e^{i m \theta}
$$

and

$$
\|v(t)\|_{L^{2}(D)}^{2}=2 \pi \sum_{m=-\infty}^{\infty}\left\|v_{m}(t)\right\|_{L^{2}\left(j^{-}, j^{+}\right)}^{2} .
$$




\subsection{The nonautonomous case: time-angle-averaging.}

The nonautonomous case when $\mathbf{u}(t, x, y)=\nabla \times \Psi(t, x, y)$ for some $T$ periodic stream-function $\Psi$ can be treated similarly as the autonomous case if the time-dependent Hamiltonian $\Psi(t)$ is completely integrable in the sense of Liouville for each $t$, and the corresponding transformation to action-angle coordinates $(x, y) \rightarrow(J, \theta)$ does not depend on $t$. In particular, $\Psi(t, x, y)=$ $\Psi(t, J)$, and introducing the frequency function as $\omega(t, J)=\partial \Psi / \partial J$, the advection-diffusion equation (1) can be written in the form

$$
c_{t}+\omega(t, J) c_{\theta}=\varepsilon(A: \nabla \nabla+\mathbf{b} \cdot \nabla) c .
$$

We write

$$
\omega(t, J)=\omega_{0}(t, J)+\omega_{1}(J),
$$

such that $\omega_{0}(\cdot, J)$ is $T$-periodic and mean-free in the time variable,

$$
\int_{0}^{T} \omega_{0}(t, J) d t=0 .
$$

An important special case is when the time-dependence is completely separable, i.e.,

$$
\Psi(t, x, y)=H(x, y) f(t)
$$

where $H$ is completely integrable in the sense of Liouville as in the previous section, and the function $f(t)=f_{0}(t)+f_{1}$ is such that $f_{0}(t)$ is $T$-periodic and mean-free function of time $t$ and $f_{1}$ is a constant. There are three dynamically distinct cases: (1) the autonomous case $\left(\omega_{0} \equiv 0\right) ;(2)$ the non-autonomous case with vanishing mean $\left(\omega_{1} \equiv 0\right)$; and $(3)$ the non-autonomous case with non-vanishing mean $\left(\omega_{0} \not \equiv 0\right.$ and $\left.\omega_{1} \not \equiv 0\right)$. We introduce

$$
\Theta(t, J)=\int_{0}^{t} \omega_{0}\left(t^{\prime}, J\right) d t^{\prime},
$$

which is also $T$-periodic in the time variable. We now use stream-lines $\bar{J}=J$ and $\bar{\theta}=\theta-\Theta(t, J)$ as new coordinates via the transformation

$$
c(t, J, \theta) \longrightarrow v(t, \bar{J}, \bar{\theta})
$$

with the following transformation rules:

$$
\begin{gathered}
c_{t}=v_{t}-v_{\bar{\theta}} \omega_{0}, \quad c_{\theta}=v_{\bar{\theta}}, \quad c_{\theta \theta}=v_{\overline{\theta \theta}}, \\
c_{J}=v_{J}-v_{\bar{\theta}} \Theta_{J}, \quad c_{J J}=v_{J J}-v_{\bar{\theta}} \Theta_{J J}-2 v_{J \bar{\theta}} \Theta_{J}+v_{\overline{\theta \theta}}\left(\Theta_{J}\right)^{2} .
\end{gathered}
$$


This transformation to stream-line coordinates is nothing else but a transformation to a new "co-moving" reference frame. Denoting by $\tilde{A}$ and $\tilde{\mathbf{b}}$ the (time-dependent) coefficient matrix and vector in these new coordinates, the equation (18) becomes

$$
v_{t}+\omega_{1}(J) v_{\bar{\theta}}=\varepsilon(\tilde{A}: \nabla \nabla+\tilde{\mathbf{b}} \cdot \nabla) v .
$$

When $\omega_{1}=0$, the advective term in equation (18) disappears, and we obtain a diffusion equation for $v$ of the form

$$
v_{t}=\varepsilon(\tilde{A}: \nabla \nabla+\tilde{\mathbf{b}} \cdot \nabla) v .
$$

All effects of the advective field are now contained in the time-dependent coefficients $\tilde{A}$ and $\tilde{\mathbf{b}}$ and, therefore, equation (22) is now suitable for averaging. This case was treated in [32] by applying a near-identity Lie transform that eliminates the explicit time dependence of the coefficients (see [32] for details). However, this approach fails in the case when $\omega_{1} \neq 0$; instead, we write the equation with time and angle averaged coefficients in an ad-hoc fashion,

$$
v_{t}+\omega_{1}(J) v_{\bar{\theta}}=\varepsilon(\langle\tilde{A}\rangle: \nabla \nabla+\langle\tilde{\mathbf{b}}\rangle \cdot \nabla) v
$$

where

$$
\left\langle\tilde{a}_{i j}\right\rangle(J)=\frac{1}{2 \pi T} \int_{0}^{T} \int_{0}^{2 \pi} \tilde{a}_{i j}(t, J, \theta) d \theta d t
$$

and

$$
\left\langle\tilde{b}_{i}\right\rangle(J)=\frac{1}{2 \pi T} \int_{0}^{T} \int_{0}^{2 \pi} \tilde{b}_{i}(t, J, \theta) d \theta d t .
$$

The convergence of solutions to the solutions of the averaged equation on timescales $T_{D}^{(\varepsilon)} \sim \varepsilon^{-1}$ will be justified in the same manner as for the autonomous case above.

\subsubsection{Unidirectional axisymmetric radial flows}

The case of unidirectional axisymmetric radial flows in annular regions or disks is very instructive both analytically and numerically. It includes physically realizable flows such as the Couette and Poiseuille flows, and the (regularized) vortical flow. More specifically, we assume that the stream function is given by

$$
\Psi(t, x, y)=H(x, y) f(t)=h(r) f(t)
$$


where $r=\sqrt{x^{2}+y^{2}}$ is the radial coordinate. For uniaxial radial flows, the action-angle variables can be expressed via the polar coordinates, $(x, y) \rightarrow$ $\left(r^{2} / 2, \theta\right)$. Denoting by $\nu(r)=h^{\prime}(r)$ the azimuthal velocity and by $\omega(r)=$ $\nu(r) / r$ the frequency, the advection-diffusion equation (1) reads in polar coordinates

$$
c_{t}+f(t) \omega(r) c_{\theta}=\varepsilon \Delta c,
$$

where $\Delta c=\left(\frac{1}{r} c_{r}+c_{r r}+\frac{1}{r^{2}} c_{\theta \theta}\right)$ is the Laplace operator in polar coordinates. In the non-autonomous case $f_{0} \not \equiv 0$, we introduce

$$
F(t)=\int_{0}^{t} f_{0}\left(t^{\prime}\right) d t^{\prime},
$$

and we derive the stream-lines equations

$$
d x / d F=\omega(r) y, \quad d y / d F=-\omega(r) x .
$$

We can now use the stream-lines $\bar{r}=r$ and $\bar{\theta}=\theta-\omega(r) F(t)$ as the new coordinates via the transformation

$$
c(t, r, \theta) \longrightarrow v(t, \bar{r}, \bar{\theta}) .
$$

Equation (21) becomes

$$
v_{t}+f_{1} \omega v_{\bar{\theta}}=\varepsilon\left(\Delta v+F\left(\left(\frac{\omega^{\prime}}{r}+\omega^{\prime \prime}\right) v_{\bar{\theta}}+2 \omega^{\prime} v_{\bar{\theta} r}\right)+F^{2}\left(\omega^{\prime}\right)^{2} v_{\overline{\theta \theta}}\right)
$$

Replacing the time-dependent coefficients by their time averages we obtain the equation

$$
V_{t}+f_{1} \omega V_{\bar{\theta}}=\varepsilon\left(\Delta V+\langle F\rangle\left(\left(\frac{\omega^{\prime}}{r}+\omega^{\prime \prime}\right) V_{\bar{\theta}}+2 \omega^{\prime} V_{\bar{\theta} r}\right)+\left\langle F^{2}\right\rangle\left(\omega^{\prime}\right)^{2} V_{\overline{\theta \theta}}\right)
$$

If instead of the above transformation we use $\bar{\theta}=\theta+\omega(r)(F(t)-\langle F\rangle)$ and denote $\operatorname{Var}(F)=\left\langle(F-\langle F\rangle)^{2}\right\rangle$, the averaged equation assumes the simpler form

$$
V_{t}+f_{1} \omega V_{\bar{\theta}}=\varepsilon\left(\Delta V+\operatorname{Var}(F)\left(\omega^{\prime}\right)^{2} V_{\overline{\theta \theta}}\right)
$$

Note that this averaged equation is essentially the autonomous advectiondiffusion equation with a small diffusive correction in the azimuthal variable, 
whose solutions converge to the solutions of the same effective diffusion equation as for the autonomous case, i.e., the equation

$$
\bar{V}_{t}=\frac{1}{r} \bar{V}_{r}+\bar{V}_{r r}
$$

The spectral boundary value problem for the autonomous non-self-adjoint operator associated with the equation (31) is the equation

$$
-\left(\varepsilon \Delta V(r, \theta)+\operatorname{Var}(F)\left(\omega^{\prime}\right)^{2} V_{\overline{\theta \theta}}\right)+\omega(r) V_{\theta}(r, \theta)=\lambda V(r, \theta)
$$

on a disk $D=\left\{0 \leq r \leq r^{+}, 0 \leq \theta \leq 2 \pi\right\}$ or an annulus $D=\left\{0<r^{-} \leq\right.$ $\left.r \leq r^{+}, 0 \leq \theta \leq 2 \pi\right\}$ subject to homogeneous Dirichlet boundary conditions $\left.V\right|_{\partial D}=0$ or the von Neumann boundary conditions $\left.\frac{\partial V}{\partial r}\right|_{\partial D}=0$. This spectral problem again allows for the separation of polar coordinates, and we seek the eigenfunctions in the form

$$
V(r, \theta)=e^{i m \theta} g_{m, n}(r),
$$

where $m$ and $n$ are integers and $g_{m, n}$ satisfies the one-dimensional spectral problem

$$
\left\langle\mathcal{L}_{m, \varepsilon}\right\rangle g_{m, n}=\lambda_{m, n} g_{m, n}
$$

with

$$
\left\langle\mathcal{L}_{m, \varepsilon}\right\rangle g=-\varepsilon\left(g^{\prime \prime}+\frac{1}{r} g^{\prime}-m^{2}\left(\frac{1}{r^{2}}+\operatorname{Var}(F)\left(\omega^{\prime}\right)^{2}\right) g\right)+i m \omega(r) g .
$$

In the case of the annulus, the boundary condition is $g_{m, n}\left(r^{ \pm}\right)=0$ (Dirichlet) or $\frac{d}{d r} g_{m, n}\left(r^{ \pm}\right)=0$ (von Neumann). Note that in the case of the disk, the singularity at $r=0$ is regular, leading to the the asymptotic behavior $g_{m, n}(r) \sim$ const $\cdot r^{m}$ as $r \rightarrow 0$. Therefore, it is plausible to impose the boundary condition $\lim _{r \rightarrow 0^{+}} \frac{g_{m, n}(r)}{r^{m}}=1$.

If $m=0,(32)$ is a self-adjoint problem,

$$
-\varepsilon\left(g_{0, n}^{\prime \prime}(r)+\frac{1}{r} g_{0, n}^{\prime}(r)\right)=\lambda_{0, n} g_{0, n}(r)
$$

Recall that in the case of the disk and the Dirichlet boundary conditions, the eigenvalue-eigenfunction pairs are $\lambda_{0, n}=\varepsilon\left(j_{0, n} / r^{+}\right)^{2}, g_{0, n}(r)=J_{0}\left(j_{0, n} r / r^{+}\right)$, where $J_{0}$ is the Bessel function and $\left\{j_{0, n}\right\}$ are its positive zeros in the increasing order. In general, the eigenfunctions are obtained in the form $g_{0, n}(r)=c_{1} H_{0}^{(1)}\left(\sqrt{\lambda_{0, n}} r\right)+c_{2} H_{0}^{(2)}\left(\sqrt{\lambda_{0, n}} r\right)$, where $H_{m}^{(1)}$ and $H_{m}^{(2)}$ are Hankel functions of order $m$. In either case, the eigenvalues scale according to $\lambda_{0, n} \sim$ const $\cdot \varepsilon$. 


\section{Non-self-adjoint Schrödinger spectral problem and WKB theory in the complex plane}

\subsection{Abel-Lidskii summation}

The purpose of this section is to study the semiclassical limit $\varepsilon \rightarrow 0$ for the non-self-adjoint 1D Schrödinger spectral problem (13). For the sake of simplicity, we assume $m=1$, and study the problem

$$
L_{\varepsilon} g_{n}=\lambda_{n} g_{n}
$$

where

$$
L_{\varepsilon} g=\left\langle\mathcal{L}_{1, \varepsilon}\right\rangle=-\varepsilon\left(\left\langle a_{11}\right\rangle(J) g^{\prime \prime}+\left\langle b_{1}\right\rangle(J) g^{\prime}-\left\langle a_{22}\right\rangle(J) g\right)+i \omega(J) g .
$$

As already noted, the spectrum consists of eigenvalues of multiplicity one, which form a denumerably infinite subset $\left\{\lambda_{n}\right\}_{n=1}^{\infty}$ of $\mathbb{C}$ having no finite points of accumulation. Moreover, the eigenfunctions $g_{n}, n \in \mathbb{N}$, form a complete set in $L^{2}\left(j^{-}, j^{+}\right)$, i.e., their span is dense in $L^{2}\left(j^{-}, j^{+}\right)$. However, the eigenfunctions (after fixing the appropriate normalization) are not orthogonal but rather bi-orthogonal,

$$
\left\langle g_{n}, \bar{g}_{k}\right\rangle=\delta_{n, k},
$$

noting that $\bar{g}_{n}, \bar{\lambda}_{n}$ are eigenfunction-eigenvalue pairs of the adjoint problem. Let us remark here that the eigenvalue-eigenfunctions pairs are denoted either $\left(\lambda_{n}^{(\varepsilon)}, g_{n}^{(\varepsilon)}\right)$, or simply $\left(\lambda_{n}, g_{n}\right)$ depending on if we need to emphasize the $\varepsilon$ dependence or not. Denoting $\rho_{n}=\Re \lambda_{n}$, we arrange the eigenvalues so that $\left\{\rho_{n}\right\}_{n=1}^{\infty}$ is a nondecreasing sequence.

For a given $g \in L^{2}\left(j^{-}, j^{+}\right)$, we formally write the spectral expansion

$$
g=\sum_{n=1}^{\infty}\left\langle g, \bar{g}_{n}\right\rangle g_{n}
$$

noting that it might not be norm-convergent in $L^{2}\left(j^{-}, j^{+}\right)$. We define the (non-orthogonal) spectral projection

$$
\mathcal{P}_{n} g=\left\langle g, \bar{g}_{n}\right\rangle g_{n},
$$

and refer to its norm $\kappa_{n}:=\left\|\mathcal{P}_{n}\right\|=\left\|g_{n}\right\|_{L^{2}}^{2}$ as the condition number of the eigenvalue $\lambda_{n}$. In general, we distinguish three distinct cases: (a) the sequence $\left\{\kappa_{n}\right\}_{n=1}^{\infty}$ is bounded, in which case the above spectral expansion 
is $L^{2}$-norm convergent; (b) the sequence $\left\{\kappa_{n}\right\}_{n=1}^{\infty}$ is polynomially bounded $\left(\kappa_{n} \leq a n^{\alpha}\right.$ for some numbers $a$ and $\left.\alpha\right)$, in which case we refer to the set $\left\{g_{n}\right\}_{n=1}^{\infty}$ as tame and we have the so-called Abel-Lidskii summation formula

$$
g=\lim _{\tau \rightarrow 0} \sum_{n=1}^{\infty} e^{-\tau n}\left\langle g, \bar{g}_{n}\right\rangle g_{n},
$$

with both limits understood in $L^{2}$-norm; (c) the sequence $\left\{\kappa_{n}\right\}_{n=1}^{\infty}$ not polynomially bounded, in which case we refer to the set $\left\{g_{n}\right\}_{n=1}^{\infty}$ as wild. We also say that the spectral problem at hand is spectrally wild. Davies et al. $[7,8]$ proved wild spectral behavior for the non-self-adjoint harmonic and anharmonic oscillators on $L^{2}(\mathbb{R})$. WKB approximations of the solutions that we will establish in the next section suggest that this is the case for more general potentials in the case of Dirichlet and von Neumann boundary conditions as well.

For a spectrally wild problem, we no longer necessarily have the AbelLidskii summation formula at our disposal; however, in many cases including the one at hand, Agranovich and Katsnelson proved [9, 10, 11] that this notion can be modified. As this result is somewhat technical to describe in all generality, here we will restrict ourselves to describing how it applies to our spectral problem. The main modification of the Abel-Lidskii summation formula lies in combining terms in the spectral expansion. More specifically, for each $\beta>0$ there exists an increasing sequence of nonnegative integers $N(r)$ depending on $\beta$, such that

$$
g=\lim _{\tau \rightarrow 0} \sum_{r=1}^{\infty} \sum_{n=N(r)+1}^{N(r+1)} e^{-\lambda_{n}^{\beta} \tau}\left\langle g, \bar{g}_{n}\right\rangle g_{n} .
$$

In the above formula, both the limit and the series are understood in $L^{2}$ metric. The main implication for us is that while $-L_{\varepsilon}$ is a generator of a strongly continuous one-parameter semigroup $e^{-L \varepsilon}$, one no longer has the $L^{2}$-convergent spectral expansion

$$
e^{-L_{\varepsilon} t} g=\sum_{n=1}^{\infty} e^{-\lambda_{n} t}\left\langle g, \bar{g}_{n}\right\rangle g_{n}
$$

However, in view of the generalized Abel-Lidskii summation formula (with $\beta=1$ ), for $\stackrel{\circ}{g} \in L^{2}\left(j^{-}, j^{+}\right)$, one has the following expansion in $L^{2}$ for the 
semigroup:

$$
e^{-L_{\varepsilon} t^{g}}=\sum_{r=1}^{\infty} \sum_{n=N(r)+1}^{N(r+1)} e^{-\lambda_{n} t}\left\langle\stackrel{\circ}{g}, \bar{g}_{n}\right\rangle g_{n} .
$$

In particular, the semi-group expansion affords a solution of the Dirichlet (von Neumann) boundary value problem

$$
g_{t}+i \omega g=\varepsilon\left(\left\langle a_{11}\right\rangle g_{J J}+\left\langle b_{1}\right\rangle g_{J}-\left\langle a_{22}\right\rangle g\right), g(0)=\stackrel{\circ}{g} .
$$

\subsection{Elements of the WKB theory}

Here, we offer a brief review of some basic facts of the WKB theory in the complex plane for second-order ordinary differential equations. A much more complete exposition can be found in [13]. The main purpose of the WKB theory is the study of the asymptotic behavior of the fundamental system of solutions for a vanishing parameter in the highest derivatives by constructing the so-called WKB approximations. Let us consider the equation

$$
w^{\prime \prime}-h^{2} q(z) w=0
$$

where $h>0$ is a parameter, and $q(z)$ is holomorphic in a domain $D$ of the complex plane. As for the WKB theory on the real line, the function

$$
S\left(z_{0}, z\right)=\int_{z_{0}}^{z} \sqrt{q(\zeta)} d \zeta
$$

is of particular importance. The WKB approximations can then be obtained in the form

$$
w_{\text {app }}^{ \pm}\left(z, h ; z_{0}\right)=q^{-1 / 4}(z) e^{ \pm h S\left(z_{0}, z\right)} .
$$

However, since $S\left(z_{0}, z\right)$ is in general a multi-valued analytic function, the validity of the WKB approximations is restricted to certain domains in the complex plane that are separated by the so-called Stokes lines.

The (anti-) Stokes lines are defined as the maximal connected analytic components of the zero level sets of the function $\Re S\left(z_{0}, z\right)\left(\Im S\left(z_{0}, z\right)\right)$. The union of all Stokes lines is called the Stokes graph, while the connected component of the Stokes graph containing the turning point (zero) $z_{0}$ of $q(z)$ is called the Stokes complex. The turning point $z_{0}$ is the branch point for the function $S\left(z, z_{0}\right)$, and the Stokes lines emanating from it are of particular interest.

If $z_{0}$ is an $n^{t h}$-order turning point, i.e., if for $z \approx z_{0}, q(z) \sim a\left(z-z_{0}\right)^{n}$ with $a \neq 0$, then

$$
S\left(z_{0}, z\right) \sim \frac{2 \sqrt{a}}{n+2}\left(z-z_{0}\right)^{\frac{n}{2}+1}
$$


and therefore there are $n+2$ Stokes lines emanating from an $n^{\text {th }}$-order turning point. In particular, three Stokes lines emanate from a simple turning point, and the angle between adjacent lines is $2 \pi / 3$.

A simply connected domain $D$ is said to be canonical if the function $S\left(z_{0}, z\right)$ is univalent on $D$. It can be easily seen that a simply connected domain contained in the complement of the Stokes graph is canonical. Moreover, $S\left(z_{0}, z\right)$ preserves sign on such canonical domains. Therefore, a simply connected domain that contains parts of one Stokes line only remains to be canonical. We refer to a canonical domain as maximal, if it is bounded by Stokes lines and is a maximal domain in which $S\left(z_{0}, z\right)$ is univalent.

We will refer to the triple $\left(D, l, z_{0}\right)$ as canonical if $l$ is a Stokes line emanating from the turning point $z_{0}$ and if $D$ is the maximal canonical domain containing $l$. The branch of $S\left(z, z_{0}\right)$ for which $\Im S\left(z, z_{0}\right)>0$ when $z \in l$ is also said to be canonical. An elementary fundamental system of solutions (FSS), $w^{ \pm}$, is uniquely determined by the canonical triple $\left(D, l, z_{0}\right)$. The validity of WKB approximations given by (37) is stated in the following theorem (see $[13])$ :

Theorem 3.1. Let $\left(D, l, z_{0}\right)$ be a canonical triple. Then the equation (36) possesses an elementary FSS, $w^{ \pm}(z, h)$, such that

$$
w^{ \pm}(z, h)=w_{\text {app }}^{ \pm}\left(z, h ; z_{0}\right)\left(1+O^{ \pm}\left(h^{-1}\right)\right)
$$

where $\left|O^{ \pm}\left(h^{-1}\right)\right| \leq C h^{-1}$ with the constant $C$ independent of $z$ as it varies on a compact set $K \subset D$. We will refer to this FSS as canonical.

Note that the validity of approximations provided by this theorem are restricted to a canonical domain, and there are several different types of transition matrices from one canonical FSS to another depending on the disposition of the canonical domains. For the purposes of this paper, we will need only the type described in the following theorem.

Theorem 3.2. Let $z_{0}$ be a simple turning point, and let $\left(D_{\text {left }}, l_{\text {left }}, z_{0}\right)$ and $\left(D_{\text {right }}, l_{\text {right }}, z_{0}\right)$ be two canonical triples such that $l_{\text {left }}$ lies to the left of $l_{\text {right }}$, and let $w_{\text {left }}^{ \pm}$and $w_{\text {right }}^{ \pm}$be the corresponding canonical FSS. Then the following transition formula is valid:

$$
\left(\begin{array}{l}
w_{\text {left }}^{+}(z) \\
w_{\text {left }}^{-}(z)
\end{array}\right)=e^{i \pi / 6}\left(\begin{array}{cc}
-i[1] & {[1]} \\
1 & 0
\end{array}\right)\left(\begin{array}{l}
w_{\text {right }}^{+}(z) \\
w_{\text {right }}^{-}(z)
\end{array}\right), \quad z \in D_{\text {right }} .
$$

Here, [1] $=1+O\left(h^{-1}\right)$, and $\left|O\left(h^{-1}\right)\right| \leq C h^{-1}$ with the constant $C$ independent of $z$ as $z$ varies on a compact set $K \subset D_{\text {right }}$. 


\subsection{Transformed problem}

In order to investigate the eigenvalue problem (13) using the complexplane WKB theory, we need to transform it into the form of equation (36). To this end, we introduce the following functions. Let $\alpha_{m}(z)$ be a solution of the equation

$$
\bar{a}_{11} \alpha_{m}^{\prime \prime}+\bar{b}_{1} \alpha_{m}^{\prime}-m^{2} \bar{a}_{22} \alpha_{m}=0,
$$

and let

$$
\beta_{m}(z):=\alpha_{m}(z)^{-2} \exp \left(\int \frac{-\bar{b}_{1}(z)}{\bar{a}_{11}(z)} d z\right) .
$$

Consider the change of variables $z \mapsto \zeta$ given through

$$
d \zeta=\beta_{m}(z) d z
$$

A straightforward calculation shows that with $h(z)=\alpha_{m}(z)^{-1} g(z)$,

$$
\bar{a}_{11}(z) g^{\prime \prime}+\bar{b}_{1}(z) g^{\prime}-m^{2} \bar{a}_{22}(z) g=\bar{a}_{11}(z) \alpha_{m}(z) \beta_{m}(z)^{2} \frac{d^{2} h}{d \zeta^{2}} .
$$

Therefore, $\lambda_{m, n}$ and $g_{m, n}$ satisfy (13) if and only if $\mu_{m, n}=-\frac{i}{m} \lambda_{m, n}$ and $h_{m, n}(\zeta)=\alpha_{m}(z)^{-1} g_{m, n}(z)$ satisfy the equation

$$
\varepsilon \bar{a}_{11} \frac{d^{2} h_{m, n}}{d \zeta^{2}}=i m \beta_{m}^{-2}\left(\omega(\zeta)-\mu_{m, n}\right) h_{m, n} .
$$

Also, denoting by $\zeta^{ \pm}=\zeta\left(j^{ \pm}\right)$, the Dirichlet boundary value problem becomes $h_{m, n}\left(\zeta^{ \pm}\right)=0$. Note that we will interchangeably refer to $\lambda_{m, n}$ and $\mu_{m, n}$ as eigenvalues, keeping the above relationship in mind.

Let us illustrate this for the case of radial flows. One can easily verify that $\alpha_{m}(r)=r^{m}, \beta_{m}(r)=r^{-(2 m+1)}$ and let $s=z^{-2 m}$. Therefore, $\lambda_{m, n}$ and $g_{m, n}$ satisfy (32) if and only if $\mu_{m, n}=-\frac{i}{m} \lambda_{m, n}$ and $h_{m, n}(s)=\frac{g_{m, n}(r)}{r^{m}}$ satisfy the equation

$$
4 m \varepsilon \frac{d^{2} h_{m, n}}{d s^{2}}=i s^{-\frac{2 m+1}{m}}\left(\omega(s)-\mu_{m, n}\right) h_{m, n} .
$$

The annulus problem is now posed on the interval $\left[s^{-}=\left(r^{+}\right)^{-2 m}, s^{+}=\right.$ $\left(r^{-}\right)^{-2 m}$, and the boundary conditions become either $h_{m, n}\left(s^{ \pm}\right)=0$ (Dirichlet) or $\left.\frac{d}{d s}\left(s^{-1 / 2} h_{m, n}(s)\right)\right|_{s=s^{ \pm}}=0$ (von Neumann). For the disk problem, the equation is now posed on $\left[s^{-}, s^{+}=+\infty\right)$, and the asymptotic behavior at the singularity leads to the boundary condition at infinity, $h_{m, n}(+\infty)=1$. 
We assume for simplicity that $\bar{a}_{11} \equiv 1$ (otherwise we would study a weighted eigenvalue problem with the weight $\bar{a}_{11}$ ). Also, we assume that $m=1$, and omit the subscripts indicating dependence on $m$. Also, let us assume that $\bar{a}_{i i}, \bar{b}_{1}$, and $\omega$ are analytic on some domain $G \subset \mathbb{C}$ containing the real interval $\left[j_{1}, j_{2}\right]$ and that the transformation $z \mapsto \zeta$ is univalent from $G$ onto some domain $\mathcal{G}$ so that $\omega$ is analytic as a function of $\zeta \in \mathcal{G}$. Then, with $q(\zeta):=i \beta(\zeta)^{-2}(\omega(\zeta)-\mu)$, we can apply the WKB theory of the previous section, with the important difference that function $S$ depends on a complex parameter $\mu$. Let $z_{\mu}$ denote the turning point of $\omega(z)-\mu$. We define

$$
S(z, \mu)=\int_{z_{\mu}}^{z} \sqrt{i(\omega(\xi)-\mu)} d \xi .
$$

Using th change of variables $z \mapsto \zeta$, we can easily check that we can identify $S(z, \mu)$ with

$$
S(\zeta, \mu)=\int_{\zeta_{\mu}}^{\zeta} \sqrt{i(\omega(\eta)-\mu)} \beta(\eta)^{-1} d \eta .
$$

For convenience, let us slightly redefine

$$
\left(L_{\varepsilon} h\right)(\zeta):=i \varepsilon \beta(\zeta)^{2} \frac{d^{2} h(\zeta)}{d \zeta^{2}}+\omega(\zeta) h(\zeta),
$$

and introduce the scalar product

$$
\left\langle h_{1}, h_{2}\right\rangle:=\int_{\zeta^{-}}^{\zeta^{+}} \beta(\zeta)^{-2} h_{1}(\zeta) h_{2}(\zeta) d \zeta .
$$

Let $\left[a^{-}, a^{+}\right]=\omega\left(\left[j^{-}, j^{+}\right]\right)$the range of $\omega$, and let

$$
\Pi=\left\{\mu \mid \Im(\mu)<0, \Re(\mu) \in\left(a^{-}, a^{+}\right)\right\}
$$

be a semi-strip in the complex plane. It is obvious that $\left\{\left\langle L_{\varepsilon} h, h\right\rangle \mid\langle h, h\rangle=\right.$ 1\} $\subset \Pi$, and hence the eigenvalues of $L_{\varepsilon}$ lie in the semi-strip $\Pi$, as well.

\subsection{The case of monotonic frequency $\omega$}

In this section, we study the Dirichlet problem (33) assuming that the frequency function $\omega:\left[j^{-}, j^{+}\right] \rightarrow\left[a^{-}, a^{+}\right]$is a strictly monotonic (w.l.o.g. increasing) function. We obtain the limit spectral graph as a tie-shaped object consisting of three analytic curves. Two of them emanate from each 
endpoint of the range of $\omega, a^{ \pm}$. They meet in a node, and are replaced by the third analytic curve that captures the eigenvalues of large modulus. We then examine the scaling laws of the eigenvalues near each branch. From those, we infer the timescales of dissipation for the solutions of the Schrödinger equation, and then the timescales of convergence of solutions of ADE to those of the effective diffusion equation.

The entire analysis presented here is carried out for the Dirichlet boundary condition. It is similar for the von Neumann boundary condition and shall be omitted.

\subsubsection{Assumptions and $W K B$ approximate solutions}

Let $\omega:\left[j^{-}, j^{+}\right] \rightarrow\left[a^{-}, a^{+}\right]$be strictly monotonic (w.l.o.g., increasing). We additionally make the following standing assumption:

Assumption 3.3. There exists a domain $G \subset \mathbb{C}$ such that $\omega: G \rightarrow \Pi$ is a univalent surjection such that $\Re\left(\frac{d}{d z} \omega\right) \geq 0$ on $G$. Furthermore, we assume that $\omega$ is continuous on $\bar{G}$ and that it is analytic on some neighborhood of the segment $\left[j^{-}, j^{+}\right]$. As above, we also assume that the change of variables $z \mapsto \zeta$ is univalent from $G$ onto some domain $\mathcal{G}$.

These assumptions will have several important consequences for the purposes of this paper:

1. $\omega:\left[j^{-}, j^{+}\right] \rightarrow\left[a^{-}, a^{+}\right]$is a strictly increasing.

2. $G$ lies entirely below the real axis.

3. For any $c \in\left(a^{-}, a^{+}\right)$, the preimage under $\omega$ of the ray $\{\mu=c-i t \mid t \geq 0\}$ is a function with respect to the imaginary axis.

4. $\omega: \mathcal{G} \rightarrow \Pi$ viewed as a function of $\zeta$ satisfies the same assumption.

5. The inverse $\omega^{-1}: \Pi \rightarrow G$ also has the property that $\Re\left(\frac{d}{d z} \omega^{-1}\right) \geq 0$ on $\Pi$.

6. For each $\mu \in \bar{\Pi}, \omega(z)-\mu$ has a unique simple turning point $z_{\mu} \in \bar{G}$.

7. As discussed in the previous subsection, for a fixed $\mu, S(z, \mu)$ is a multivalued function that is locally analytic with respect to $z \in G$ with the branch point $z_{\mu}$. There are three Stokes lines emanating from of the turning point $z_{\mu}$. With respect to the vertical line at $z_{\mu}$, we will label them as the 'left', $\ell_{\mu}^{\text {left }}$, the 'right', $\ell_{\mu}^{\text {right }}$, and the 'lower', $\ell_{\mu}^{\text {lower }}$. We identify three maximal canonical domains, each of which has one of the Stokes lines from the Stokes complex belonging to it, while the other two (and possibly others not belonging to this Stokes complex) lie on 
its boundary. We denote each one of these domains by $\Omega_{\mu}^{\text {left }}, \Omega_{\mu}^{\text {right }}$ or $\Omega_{\mu}^{\text {lower }}$, depending on which one of the three Stokes lines belongs to it. Note that $S(z, \mu)$ can be extended analytically on either but not simultaneously on both sides of the Stokes lines on its boundary. (See Figure 1)

8. $S(z, \mu)$ is analytic on $\Pi$ and continuous on $\bar{\Pi}$ with respect to the variable $\mu$. For $\mu=c \in\left(a^{-}, a^{+}\right)$, we fix the branch so that $\Re S\left(j^{+}, c\right)>0$. For other values of $\mu \in \bar{\Pi}$, the branch is fixed so that $S\left(j^{+}, \mu\right)$ and $S\left(j^{+}, c\right)$ are analytically connected. We proceed similarly for $S\left(j^{-}, \mu\right)$.

As discussed in the previous subsection, from the general WKB theory applied to the equation (39), we know that it possesses two so-called WKB approximations of the form

$$
h_{\mathrm{app}}^{ \pm}(\zeta, \mu)=\frac{\beta(\zeta)^{1 / 2}}{\sqrt[4]{i(\tilde{\omega}(\zeta)-\mu)}} e^{ \pm \varepsilon^{-\frac{1}{2}} S(\zeta, \mu)},
$$

which lead to the WKB approximations of (13)

$$
g_{\text {app }}^{ \pm}(z, \mu)=\frac{\beta(z)^{1 / 2} \alpha(z)}{\sqrt[4]{i(\omega(z)-\mu)}} e^{ \pm \varepsilon^{-\frac{1}{2}} S(z, \mu)} .
$$

Note that for radial flows, the formula reads

$$
g_{\text {app }}^{ \pm}(r, \mu)=\frac{r^{-1 / 2}}{\sqrt[4]{i(\omega(r)-\mu)}} e^{ \pm \varepsilon^{-\frac{1}{2}} S(r, \mu)} .
$$

The following theorem is the analogue of the Theorem 3.1 on the validity of WKB approximations in this particular situation. We will use the Birkhoff notation $[1]^{ \pm}=1+O^{ \pm}\left(\varepsilon^{\frac{1}{2}}\right)$.

Theorem 3.4. Let $\mu \in \Pi$, and let $\left(\Omega_{\mu}, l_{\mu}, z_{\mu}\right)$ be a canonical triple associated with the turning point $z_{\mu}$. equation (39) possesses two linearly independent solutions of the form

$$
g^{ \pm}(z, \mu)=g_{\mathrm{app}}^{ \pm}(z, \mu)\left(1+O^{ \pm}\left(\varepsilon^{\frac{1}{2}}\right)\right),
$$

where $O^{ \pm}$satisfies $\left|O^{ \pm}\left(\varepsilon^{\frac{1}{2}}\right)\right| \leq C \varepsilon^{\frac{1}{2}}$, with a constant $C$ not depending on $z$ as it varies on a compact set $K$ belonging to a canonical domain $\Omega_{\mu}$. Moreover, the constant $C$ does not depend on $\mu$ or $z$ as they vary on compact sets $K^{\prime} \subset \bar{\Pi}$ and $K \subset \cap_{\mu \in K^{\prime}} \Omega_{\mu}$, respectively. 


\subsubsection{Spectral graph}

We now introduce several functions that depend solely on the parameter $\mu$ that determine the spectral graph of the eigenvalue problem. While related, these functions are not to be confused with the function $S(z, \mu)$ used in the construction of the WKB approximations. Similarly, the tie-shaped spectral graph (in Figure 2) is not to be confused with the three-line Stokes complex described in the previous sections (in Figure 1). We define

$$
Q^{ \pm}(\mu)= \pm S\left(j^{ \pm}, \mu\right)= \pm \int_{z_{\mu}}^{j^{ \pm}} \sqrt{i(\omega(\xi)-\mu)} d \xi
$$

Also,

$$
Q^{\infty}(\mu)=\int_{j^{-}}^{j^{+}} \sqrt{i(\omega(\xi)-\mu)} d \xi
$$

where the variable of integration $\xi \in\left[j^{-}, j^{+}\right]$. Note that the definition would suggest that $Q^{\infty}(\mu)=S\left(j^{+}, \mu\right)-S\left(j^{-}, \mu\right)=Q^{+}(\mu)+Q^{-}(\mu)$, however this is only true if the segment $\left[j^{-}, j^{+}\right]$is contained within a canonical domain. Recall that we fixed the branches by the condition that for $c \in\left(a^{-}, a^{+}\right)$, $Q^{+}(c)=e^{i \pi / 4} \alpha_{c}^{+}$with $\alpha_{c}^{+}>0$ and $Q^{-}(c)=e^{i \pi / 4} \alpha_{c}^{-}$with $\alpha_{c}^{-}<0$. Let us define the level sets

$$
\tilde{\gamma}_{ \pm}=\left\{\mu \in \bar{\Pi} \mid \Re Q^{ \pm}(\mu)=0\right\} \text { and } \tilde{\gamma}_{\infty}=\left\{\mu \in \bar{\Pi} \mid \Re Q^{\infty}(\mu)=0\right\} .
$$

In the next theorem, we summarize some important properties of these functions.

Lemma 3.5. For $\mu \in \Pi$, we have $\Im \frac{d}{d \mu} Q^{+}(\mu)<0$, $\Im \frac{d}{d \mu} Q^{-}(\mu)>0$ and $\Re \frac{d}{d \mu} Q^{\infty}(\mu)<0$. Consequently, the functions $Q^{+}(\mu), Q^{-}(\mu)$ and $Q^{\infty}(\mu)$ are univalent in the semi-strip $\Pi$.

Proof Let $\mu=c-i t$ for some $c \in\left(a^{-}, a^{+}\right)$and $t>0$. Let $z_{c} \in\left(j^{-}, j^{+}\right)$be the turning point of $\omega(\mu)-c$. We write

$$
Q^{+}(\mu)=\int_{z_{\mu}}^{z_{c}} \sqrt{i(\omega(\xi)-\mu)} d \xi+\int_{z_{c}}^{j^{+}} \sqrt{i(\omega(\xi)-\mu)} d \xi=: Q_{1}^{+}(\mu)+Q_{2}^{+}(\mu) .
$$

Clearly, for $\xi \in\left(z_{c}, j^{+}\right)$, $\arg (\omega(\xi)-c+i t) \in(0, \pi / 2)$, and

$$
\Im\left(\frac{d}{d \mu} Q_{2}^{+}(\mu)\right)=-\frac{1}{2} \int_{z_{c}}^{j^{+}} \Im\left(\frac{e^{i \pi / 4}}{\sqrt{(\omega(\xi)-c)+i t}}\right) d \xi<0 .
$$


By property 3 of the frequency $\omega$, the path $\omega^{-1}(c-i \infty, c]$ in $G$ allows for the parametrization of the form $\omega^{-1}(c-i \tau)=\rho(\sigma(\tau))-i \sigma(\tau), 0 \leq \tau<\infty$, with $d \sigma / d \tau \geq 0$. Therefore,

$$
Q_{1}^{+}(\mu)=\int_{z_{\mu}}^{z_{c}} \sqrt{i(\omega(\xi)-\mu)} d \xi=-\int_{0}^{t} \sqrt{t-\tau} d(\sigma(\tau)+i \rho(\sigma(\tau)) .
$$

Therefore, $\frac{\partial}{\partial t} \Re Q_{1}^{+}(c-i t)<0$, and consequently $\Im \frac{d}{d \mu} Q_{1}^{+}(\mu)<0$. The statement for $Q^{-}$follows similarly. Observe that since for $\xi \in\left(j^{-}, j^{+}\right)$, $\arg (\omega(\xi)-\mu) \in(0, \pi)$, we have

$$
\Re\left(\frac{d}{d \mu} Q^{\infty}(\mu)\right)=-\frac{1}{2} \int_{j^{-}}^{j^{+}} \Re\left(\frac{e^{i \pi / 4}}{\sqrt{\omega(\xi)-\mu}}\right) d \xi<0 .
$$

Theorem 3.6. The curves $\tilde{\gamma}_{-}$and $\tilde{\gamma}_{+}$are one-to-one with respect to the interval $\left[a^{-}, a^{+}\right]$and they pass through the points $a^{-}$and $a^{+}$, respectively. The curve $\tilde{\gamma}_{\infty}$ is one-to-one with respect to $[0,-i \infty)$ on the imaginary axis. The function $\Im Q^{+}(\mu)\left(\Im Q^{-}(\mu)\right.$ ) is strictly decreasing (increasing) on $\tilde{\gamma}_{+}\left(\tilde{\gamma}_{-}\right)$ with respect to the real axis. The function $\Im Q^{\infty}(\mu)$ is strictly decreasing on $\tilde{\gamma}_{\infty}$ with respect to the imaginary axis. The function $\Re Q^{+}(\mu)\left(\Re Q^{-}(\mu)\right)$ is positive (negative) above the curve $\tilde{\gamma}_{+}\left(\tilde{\gamma}_{-}\right)$, and it is of the opposite sign bellow that curve. The three curves have a unique intersection point (knot) $\mu_{0}$.

Proof It is obvious that $a^{-} \in \tilde{\gamma}_{-}$. In the previous lemma, we have proven that for $c \in\left(a^{-}, a^{+}\right)$the function $\Re Q^{+}(c-i t)$ decreases with $t$. Since $\Re Q^{+}(c)>0$ and $\lim _{t \rightarrow \infty} \Re Q^{+}(c-i t)<0, t \mapsto \Re Q^{+}(c-i t)$ has a unique root $T_{C}^{(\varepsilon)}>0$. Since $\Im \frac{d}{d \mu} Q^{+}(\mu)<0$, the function $\Im Q^{+}(\mu)$ is strictly decreasing on $\tilde{\gamma}_{+}$with respect to the real axis. The proof of the statement for $Q^{-}$is similar.

As for $Q^{\infty}$, note that

$$
\Re Q^{\infty}\left(a^{-}-i t\right)=\Re \int_{a^{-}}^{a^{+}} \sqrt{i\left(\omega(\xi)-a^{-}\right)+i t} d \xi>0
$$

and

$$
\Re Q^{\infty}\left(a^{+}-i t\right)=\Re \int_{a^{-}}^{a^{+}} \sqrt{i\left(\omega(\xi)-a^{+}\right)+i t} d \xi<0,
$$


and since for fixed $t>0$ the function $c \mapsto \Re Q^{\infty}(c-i t)$ is strictly decreasing, it has a unique root $c_{t} \in\left(a^{-}, a^{+}\right)$. Since $\Re \frac{d}{d \mu} Q^{\infty}(\mu)<0$, the function $\Im Q^{\infty}(\mu)$ is strictly decreasing on $\tilde{\gamma}_{\infty}$ with respect to the imaginary axis.

Clearly, the curves $\tilde{\gamma}_{-}$and $\tilde{\gamma}_{+}$have at least one intersection point $\mu_{0}$. Let

$$
Q_{0}(\mu)=\int_{z_{\mu_{0}}}^{z_{\mu}} \sqrt{i(\omega(\xi)-\mu)} d \xi=Q^{+}\left(\mu_{0}\right)-Q^{+}(\mu)=Q^{-}(\mu)-Q^{-}\left(\mu_{0}\right) .
$$

$Q_{0}$ is analytic on $\Pi$, and hence $\Re Q_{0}$ is harmonic and $\tilde{\gamma}_{-} \cup \tilde{\gamma}_{+}$is contained in its zero level set. If there were any other intersection points between $\tilde{\gamma}_{-}$and $\tilde{\gamma}_{+}$, by maximum principle $\Re Q_{0}$ would be identical to zero on $\Pi$, which is a contradiction. It is obvious that $\mu_{0}$ also lies on $\tilde{\gamma}_{\infty}$.

Theorem 3.6 implies that the lines $\tilde{\gamma}_{+}$and $\tilde{\gamma}_{-}$divide $\Pi$ into four regions: $\Pi^{\text {upper }}$ (above both $\tilde{\gamma}_{+}$and $\tilde{\gamma}_{-}$), $\Pi^{\text {lower }}$ (below both $\tilde{\gamma}_{+}$and $\tilde{\gamma}_{-}$), $\Pi^{\text {left }}$ (above $\tilde{\gamma}_{+}$and below $\tilde{\gamma}_{-}$) and $\Pi^{\text {right }}$ (above $\tilde{\gamma}_{-}$and below $\tilde{\gamma}_{+}$). We denote by $\gamma_{+}, \gamma_{-}$ and $\gamma_{\infty}$ the parts of the curves $\tilde{\gamma}_{-}, \tilde{\gamma}_{+}$and $\tilde{\gamma}_{\infty}$ between the knot $\mu_{0}$ and the points $a^{-}, a^{+}$and $-i \infty$ respectively (see Figure 2). Let $\Gamma=\gamma_{-} \cup \gamma_{+} \cup \gamma_{\infty}$. We will prove that the set $\Gamma$ is the semiclassical limit spectral graph of the Dirichlet problem (13) in the sense that it is the set of accumulation points of the Dirichlet eigenvalues of (13) as $\varepsilon \rightarrow 0$. The following lemma will be crucial in the proof of that statement.

Lemma 3.7. For each $\mu \in \Pi$, no Stokes lines other than $\ell_{\mu}^{\text {left }}$ and $\ell_{\mu}^{\text {right }}$ intercept the segment $\left[j^{-}, j^{+}\right]$. In addition, we have

1. If $\mu \in \tilde{\gamma}_{-}$, then $\ell_{\mu}^{\text {left }} \cap\left[j^{-}, j^{+}\right]=\left\{j^{-}\right\}$

2. If $\mu \in \tilde{\gamma}_{+}$, then $\ell_{\mu}^{\text {right }} \cap\left[j^{-}, j^{+}\right]=\left\{j^{+}\right\}$

3. If $\mu \in \Pi^{\text {upper }} \cup \Pi^{\text {left }} \cup \gamma^{-}$then $\ell_{\mu}^{\text {right }} \cap\left(j^{-}, j^{+}\right)=\left\{z^{+}(\mu)\right\}$

4. If $\mu \in \Pi^{\text {upper }} \cup \Pi^{\text {right }} \cup \gamma^{+}$then $\ell_{\mu}^{\text {left }} \cap\left(j^{-}, j^{+}\right)=\left\{z^{-}(\mu)\right\}$

5. If $\mu \in \Pi^{\text {left }} \cup \Pi^{\text {lower }} \cup\left(\tilde{\gamma}_{+} \backslash \gamma_{+}\right)$then $\ell_{\mu}^{\text {left }} \cap\left(j^{-}, j^{+}\right)=\emptyset$

6. If $\mu \in \Pi^{\text {right }} \cup \Pi^{\text {lower }} \cup\left(\tilde{\gamma}_{-} \backslash \gamma_{-}\right)$then $\ell_{\mu}^{\text {right }} \cap\left(j^{-}, j^{+}\right)=\emptyset$.

In particular, the points $j^{-}$and $j^{+}$both belong to a canonical domain associated with $\mu \in \Pi$ if and only if $\mu \notin \gamma_{+} \cup \gamma_{-}$.

Proof For $\mu=c \in\left(a^{-}, a^{+}\right)$, the Stokes complex associated with $S(z, c)$ has a turning point at $z_{c} \in\left(j^{-}, j^{+}\right)$, and three lines emanating from it. Because of the continuity, for sufficiently small $t>0$, the turning point associated with 
$S(z, \mu)$ with $\mu=c-i t$ belongs to $\Pi$, and the lines $\ell_{\mu}^{\text {left }}$ and $\ell_{\mu}^{\text {right }}$ intercept the segment $\left(j^{-}, j^{+}\right)$at two points $z^{-}(t)<z^{+}(t)$ respectively, and they depend continuously on $t$. For $z \in\left(z^{+}(t), j^{+}\right)$we have

$$
\Re S(z, c-i t)=\Re \int_{z_{\mu}}^{z^{+}(t)} \sqrt{i(\omega(\xi)-\mu)} d \xi+\Re \int_{z^{+}(t)}^{z} \sqrt{i(\omega(\xi)-\mu)} d \xi>0,
$$

since the point $z^{+}$lies on a Stokes line. Therefore, there are no Stokes lines that intercept $\left(z^{+}, j^{+}\right)$. Similar arguments lead to the same conclusion on intervals $\left(j^{-}, z^{-}\right)$and $\left(z^{-}, z^{+}\right)$. Further, we have shown that $t \mapsto Q^{+}(c-i t)$ is a decreasing function, and since

$$
Q^{+}(c-i t)=\Re S\left(j^{+}, c-i t\right)=\Re \int_{z^{+}(t)}^{j^{+}} \sqrt{i(\omega(\xi)-\mu)} d \xi
$$

$z^{+}(t)$ is an increasing function that attains the value $j^{+}$for $c-i t \in \tilde{\gamma}_{+}$. Similar arguments hold for $z^{-}(t)$, and the assertions 1-6 follow.

If $\mu \in \Pi^{\text {left }}$, then $j^{-}, j^{+} \in \Omega_{\mu}^{\text {right }}$ and if $\mu \in \Pi^{\text {right }}$, then $j^{-}, j^{+} \in \Omega_{\mu}^{\text {left }}$. If $\mu \in \Pi^{\text {lower }}$, then $j^{-}, j^{+} \in \Omega_{\mu}^{\text {right }} \cap \Omega_{\mu}^{\text {right }}$. If $\mu \in \Pi^{\text {upper }}$, then $j^{-}, j^{+} \in \Omega_{\mu}^{\text {lower }}$.

The next theorem is the first step in establishing $\Gamma$ as the set of accumulation points of eigenvalues as $\varepsilon \rightarrow 0$.

Theorem 3.8. Given $\tau>0$ there exists $\varepsilon_{0}>0$ such that all the Dirichlet eigenvalues of equation (13) lie in the $\tau$-neighborhood $\Gamma_{\tau}$ of $\Gamma$ provided that $0<\varepsilon<\varepsilon_{0}$.

Proof For a given $\mu$, a nontrivial linear combination of the fundamental set of solutions (43), $g(z)=c_{1} g^{-}(z, \mu)+c_{2} g^{+}(z, \mu)$, satisfies the Dirichlet boundary condition $g\left(j^{+}, \mu\right)=g\left(j^{-}, \mu\right)=0$ if and only if the characteristic determinant,

$$
\Delta(\mu)=\left|\begin{array}{ll}
g^{+}\left(j^{+}, \mu\right) & g^{+}\left(j^{-}, \mu\right) \\
g^{-}\left(j^{+}, \mu\right) & g^{-}\left(j^{-}, \mu\right)
\end{array}\right|
$$

does not vanish. Therefore, it suffices to show that there exists $\varepsilon_{0}>0$ so that for $0<\varepsilon<\varepsilon_{0}, \Delta(\mu) \neq 0$ for $\mu \in \Pi \backslash \Gamma_{\tau}$. The set $\Pi \backslash \Gamma_{\tau}$ consists of three disjoint, connected, closed components, the 'left', $\Lambda_{l}$, the 'right', $\Lambda_{r}$ and the 'upper', $\Lambda_{u}$. By virtue of the previous lemma, for $\mu \in \Pi \backslash\left(\gamma_{-} \cup \gamma_{+}\right)$there 
exists a canonical domain $\Omega_{\mu}$ containing the points $j^{-}$and $j^{+}$. Hence, the following representation holds:

$$
\Delta(\mu)=T(\mu)\left(e^{\varepsilon^{-\frac{1}{2}}\left(S\left(j^{+}, \mu\right)-S\left(j^{-}, \mu\right)\right)}[1]-e^{-\varepsilon^{-\frac{1}{2}}\left(S\left(j^{+}, \mu\right)-S\left(j^{-}, \mu\right)\right)}[1]\right)
$$

where

$$
T(\mu)=\left(i\left(\omega\left(j^{-}\right)-\mu\right)\right)^{-1 / 4}\left(i\left(\omega\left(j^{+}\right)-\mu\right)\right)^{-1 / 4}\left(\beta\left(j^{-}\right) \beta\left(j^{+}\right)\right)^{1 / 2} \alpha\left(j^{-}\right) \alpha\left(j^{+}\right),
$$

which does not vanish in $\Pi$. Therefore, $\Delta(\mu)=0$ is only possible if

$$
e^{ \pm \varepsilon^{-\frac{1}{2}}\left(S\left(j^{+}, \mu\right)-S\left(j^{-}, \mu\right)\right)}=1+O\left(\varepsilon^{\frac{1}{2}}\right) .
$$

A sufficient condition for $\Delta(\mu) \neq 0$ is therefore $\left|\Re\left(S\left(j^{+}, \mu\right)-S\left(j^{-}, \mu\right)\right)\right|>$ $C(\tau)$ with a constant $C(\tau)$ depending only on $\tau$. The proof requires a separate discussion depending on the position of $\mu$ with respect to the lines $\tilde{\gamma}_{ \pm}$. In the case when $\mu$ is above $\left(\tilde{\gamma}_{-} \backslash \gamma_{-}\right) \cup\left(\tilde{\gamma}_{+} \backslash \gamma_{+}\right)$, we use the fact that $j^{-}$and $j^{+}$are connected by a canonical path $\gamma_{\mu}$ belonging to $\Omega_{\mu}$ which intercepts one Stokes line only, at which $\Re S(r, \mu)$ changes sign. Therefore, $\Re S\left(j^{+}, \mu\right)$ and $\Re S\left(j^{-}, \mu\right)$ are of opposite signs. Straightforward compactness arguments yield the desired conclusion. For $\mu$ on or under $\left(\tilde{\gamma}_{-} \backslash \gamma_{-}\right) \cup\left(\tilde{\gamma}_{+} \backslash \gamma_{+}\right), \gamma_{\mu}$ can be chosen to be the segment $\left[j^{-}, j^{+}\right]$, and therefore w.l.o.g., $S\left(j^{+}, \mu\right)-S\left(j^{-}, \mu\right)=$ $Q^{\infty}(\mu) . S\left(j^{+}, \mu\right)$ and $S\left(j^{-}, \mu\right)$ are of the same sign; however, we make use of the fact that $\Lambda_{l} \cup \Lambda_{r}$ is order of $\tau$ distance from the set where $\Re\left(Q^{\infty}(\mu)\right)=0$ to arrive at the same conclusion. Note that $\Delta(\mu) \neq 0$ in this case follows from the condition

$$
e^{ \pm \varepsilon^{-\frac{1}{2}} Q^{\infty}(\mu)}=1+O\left(\varepsilon^{\frac{1}{2}}\right) .
$$

Further details can be found in [30].

The second step in establishing $\Gamma$ as the limit spectral graph involves showing that for each $\mu \in \Gamma$ and a neighborhood $U$ of $\mu$, there exist an Dirichlet eigenvalue $\mu(\varepsilon) \in U$ of equation (13). This will be accomplished by providing approximations of the eigenvalues that involve functions $Q^{ \pm}$and $Q^{\infty}$. More specifically, for $k \in \mathbb{N}$ and $\varepsilon>0$, let us define the points $\mu_{k}^{+}(\varepsilon) \in \tilde{\gamma}_{+}$, $\mu_{k}^{-}(\varepsilon) \in \tilde{\gamma}_{-}$and $\mu_{k}^{\infty}(\varepsilon) \in \tilde{\gamma}_{\infty}$ as the respective solutions of the equations

$$
\begin{array}{r}
\Im Q^{+}(\mu)=\varepsilon^{\frac{1}{2}}(k \pi-\pi / 4), \\
\Im Q^{-}(\mu)=\varepsilon^{\frac{1}{2}}(k \pi-\pi / 4) \\
\Im Q^{\infty}(\mu)=\varepsilon^{\frac{1}{2}} k \pi .
\end{array}
$$


The first two definitions make sense up to some $k=q_{+}^{\prime}\left(k=q_{-}^{\prime}\right)$ because, as we established before, $\Im Q^{+}(\mu)\left(\Im Q^{-}(\mu)\right)$ is strictly decreasing (increasing) on $\tilde{\gamma}_{+}\left(\tilde{\gamma}_{-}\right)$with respect to the real axis. The latter definition makes sense for all $k \in \mathbb{N}$, since the function $\Im Q^{\infty}(\mu)$ is strictly decreasing on $\tilde{\gamma}_{\infty}$ with respect to the imaginary axis.

Theorem 3.9. Let $\delta>0$ and let $\left\{\mu_{k}^{+}\right\}_{p_{+}}^{q_{+}},\left\{\mu_{k}^{-}\right\}_{p_{-}^{-}}^{q_{-}}$, and $\left\{\mu_{k}^{\infty}\right\}_{p_{\infty}}^{\infty}$ denote those points which lie on $\gamma_{+}, \gamma_{-}$and $\gamma_{\infty}$ but outside $U_{\delta}\left(a^{-}\right) \cup U_{\delta}\left(a^{+}\right) \cup U_{\delta}\left(\mu_{0}\right)$. Then there exists $C=C(\delta)>0$ such that every Dirichlet eigenvalue of equation (39) lies either in $U_{\delta}\left(a^{-}\right) \cup U_{\delta}\left(a^{+}\right) \cup U_{\delta}\left(\mu_{0}\right)$ or in a $C \varepsilon$ neighborhood of one of the points from those three sets. Each neighborhood contains exactly one eigenvalue.

Proof From the previous theorem, we know that the eigenvalues lie arbitrarily close to one of the sets $\gamma_{+}, \gamma_{-}$and $\gamma_{\infty}$. Let us first focus on the set $\gamma_{+}$and let us for the sake of clarity assume that $\mu \in \gamma_{+}^{\prime}:=\gamma_{+} \backslash\left(U_{\delta}\left(a^{+}\right) \cup\right.$ $\left.U_{\delta}\left(\mu_{0}\right)\right)$. Recall that with each $\mu \in \Pi$ we associate a three-line Stokes complex $\mathcal{C}_{\mu}=\ell_{\mu}^{\text {left }} \cup \ell_{\mu}^{\text {right }} \cup \ell_{\mu}^{\text {lower }}$. Let us consider the two sets of fundamental solutions $g_{\text {left }}^{ \pm}$and $g_{\text {right }}^{ \pm}$associated with canonical domains $\Omega_{\mu}^{\text {left }}$ and $\Omega_{\mu}^{\text {right }}$. We have established that $j^{+} \in \Omega_{\mu}^{\text {right }}$ and $j^{-} \in \Omega_{\mu}^{\text {left }}$. For $\mu \in \gamma_{+}$, we have $j^{+} \in \ell_{\mu}^{\text {right }}$. In the expression (43) for $g_{\text {left }}^{ \pm}$, we fix a branch by the condition $\Re S\left(j^{-}, \mu\right)=\alpha(\mu)<\alpha$ for $\mu$ sufficient close to $\gamma_{+}^{\prime}$ for some $\alpha<0$. We can also choose $\tau>0$, so that $\Re S\left(j^{+}, \mu\right)<\alpha(\mu) / 2$ for $\mu$ within $\tau$-distance of $\gamma_{+}^{\prime}$ and outside of $U_{\delta}\left(a^{+}\right) \cup U_{\delta}\left(\mu_{0}\right)$. Consequently, $\exp \left[\varepsilon^{-\frac{1}{2}}\left(S\left(j^{-}, \mu\right)+S\left(j^{+}, \mu\right)\right)\right]$ decays exponentially as $\varepsilon \rightarrow 0$ for such $\mu$ as well.

The two fundamental sets of solutions (43) are related through the following transition formula for neighboring canonical domains:

$$
\left(\begin{array}{c}
g_{\text {left }}^{+}(z, \mu) \\
g_{\text {left }}^{-}(z, \mu)
\end{array}\right)=e^{i \pi / 6}\left(\begin{array}{cc}
-i[1] & {[1]} \\
1 & 0
\end{array}\right)\left(\begin{array}{c}
g_{\text {right }}^{+}(z, \mu) \\
g_{\text {right }}^{-}(z, \mu)
\end{array}\right), \quad z \in \Omega_{\mu}^{\text {right }} .
$$

Here, as before $[1]=1+O\left(\varepsilon^{\frac{1}{2}}\right)$ and $\left|O\left(\varepsilon^{\frac{1}{2}}\right)\right| \leq C \varepsilon^{\frac{1}{2}}$. The constant $C=C(z, \mu)$ depends on $z$ and $\mu$ in general. However, for a given compact set $K$ in $\Omega_{\mu}^{\text {right }}$ there exists a neighborhood $U(\mu)$ of $\mu$ such that $C=C(K)$ depends only on $K$.

Consider again the characteristic determinant

$$
\Delta(\mu)=\left|\begin{array}{cc}
g_{\text {left }}^{+}\left(j^{-}, \mu\right) & g_{\text {left }}^{+}\left(j^{+}, \mu\right) \\
g_{\text {left }}^{-}\left(j^{-}, \mu\right) & g_{\text {left }}^{-}\left(j^{+}, \mu\right)
\end{array}\right| .
$$


Using the transition formula, we obtain

$$
\Delta(\mu)=T(\mu) e^{i \pi / 6}\left|\begin{array}{cc}
{[1] e^{\varepsilon^{-\frac{1}{2}} S\left(j^{-}, \mu\right)}} & -i[1] e^{\varepsilon^{-\frac{1}{2}}\left(S\left(j^{+}, \mu\right)\right.}+[1] e^{-\varepsilon^{-\frac{1}{2}} S\left(j^{+}, \mu\right)} \\
{[1] e^{-\varepsilon^{-\frac{1}{2}} S\left(j^{-}, \mu\right)}} & {[1] e^{\varepsilon^{-\frac{1}{2}} S\left(j^{+}, \mu\right)}}
\end{array}\right|
$$

As $\varepsilon \rightarrow 0$, the term $\exp \left[\varepsilon^{-\frac{1}{2}}\left(S\left(j^{-}, \mu\right)+S\left(j^{+}, \mu\right)\right)\right]$ decays exponentially, while $\exp \left(-\varepsilon^{-\frac{1}{2}} S\left(j^{-}, \mu\right)\right)$ decays exponentially and $\exp \left(\varepsilon^{-\frac{1}{2}} S\left(j^{+}, \mu\right)\right)$ remains bounded. Therefore, $\Delta(\mu)=0$ is equivalent up to exponentially small terms to the equation

$$
[1] e^{-\varepsilon^{-\frac{1}{2}} S\left(j^{+}, \mu\right)}-i[1] e^{\varepsilon^{-\frac{1}{2}} S\left(j^{+}, \mu\right)}=0 .
$$

A necessary condition for the latter equation is

$$
\cos \left(\varepsilon^{-\frac{1}{2}} \Im Q^{+}(\mu)-\frac{\pi}{4}\right)=O\left(\varepsilon^{\frac{1}{2}}\right) .
$$

The unperturbed equation (obtained by neglecting the $O\left(\varepsilon^{\frac{1}{2}}\right)$ term) leads to

$$
\Im Q^{+}(\mu)=\varepsilon^{\frac{1}{2}}(k \pi-\pi / 4), \quad k \in \mathbb{N} .
$$

The existence of simple roots of the perturbed equation in $C \varepsilon$ neighborhoods of $\mu_{k}^{+}(\varepsilon)$ follows by applying standard arguments based on Rouché's theorem.

Near $\gamma_{\infty}$, the conclusion is simpler and does not require the transition formula. Note that the relation (45) for eigenvalues bellow $\left(\tilde{\gamma}_{-} \backslash \gamma_{-}\right) \cup\left(\tilde{\gamma}_{+} \backslash \gamma_{+}\right)$ reads

$$
\sin \left(\varepsilon^{-\frac{1}{2}} \Im Q^{\infty}(\mu)\right)=O\left(\varepsilon^{\frac{1}{2}}\right) .
$$

Neglecting $O\left(\varepsilon^{\frac{1}{2}}\right)$, we obtain the following formula for the solutions

$$
\Im Q^{\infty}(\mu)=\varepsilon^{\frac{1}{2}} k \pi, \quad k \in \mathbb{Z} .
$$

Further details can be found in [29, 30].

Remark The last two theorems remain valid in the case of the disk. The proof has to be slightly modified, however, because the approximation (43) breaks down in the neighborhood of the singularity $r=0$. Instead, for $r \ll 1$, (43) can be replaced by the following approximation

$$
g_{\mathrm{app}}^{ \pm}(r, \mu)=\frac{r^{m}}{\sqrt[4]{i(\omega(r)-\mu)}} e^{ \pm(m \varepsilon)^{-\frac{1}{2}} S(r, \mu)} .
$$

Recall that the boundary condition at $r=0$ is set to $\lim _{r \rightarrow 0^{+}} \frac{g_{m, n}(r)}{r^{m}}=1$. The discussion about the sign of the characteristic determinant is the same as in the case of the annulus. 


\subsubsection{Sublinear scaling of Dirichlet eigenvalues}

In this subsection, we are investigating the scaling of the Dirichlet eigenvalues of equation (13) with respect to the vanishing diffusivity, $\varepsilon \rightarrow 0$. We first show that $\mu_{k}^{ \pm}(\varepsilon)-a^{ \pm}$scale sublinearly with $\varepsilon$, i.e., $\mu_{k}^{ \pm}(\varepsilon)-a^{ \pm} \sim$ const. $\cdot \varepsilon^{\alpha}$ as $\varepsilon \rightarrow 0$ for some $\alpha \in(0,1)$. Recall that $\mu_{k}^{ \pm}(\varepsilon) \rightarrow a^{ \pm}$(and in particular $\left.\Im \mu_{k}^{ \pm}(\varepsilon) \rightarrow 0\right)$ as $\varepsilon \rightarrow 0$.

The function $Q^{-}$can be computed explicitly for $\omega(j)=a^{-}+\left(j-j^{-}\right)$and $\omega(j)=a^{-}+\left(j-j^{-}\right)^{2}$. For the former, straightforward integration leads to the formula

$$
Q^{ \pm}(\mu)= \pm \frac{2 e^{i \pi / 4}}{3}\left(a^{ \pm}-\mu\right)^{\frac{3}{2}}
$$

Introducing the notation $j_{k}=\left(\frac{3 \pi}{2}\left(k-\frac{1}{4}\right)\right)^{\frac{2}{3}}$, we obtain

$$
\mu_{k}^{ \pm}(\varepsilon)=a^{ \pm} \mp e^{ \pm i \pi / 6} \varepsilon^{\frac{1}{3}} j_{k}
$$

hence, $\mu_{k}^{ \pm}(\varepsilon)-a^{ \pm} \sim$ const. $\cdot \varepsilon^{\frac{1}{3}}$ as $\varepsilon \rightarrow 0$.

In the second case, integration yields the formula

$$
Q^{-}(\mu)=\frac{\pi}{4} e^{3 \pi / 4}\left(\mu-a^{-}\right)
$$

hence,

$$
\mu_{k}^{-}(\varepsilon)=a^{-}+(1-4 k) \varepsilon^{\frac{1}{2}} e^{-i \pi / 4} .
$$

In this case, $\mu_{k}^{-}(\varepsilon)-a^{-} \sim$ const. $\cdot \varepsilon^{\frac{1}{2}}$ as $\varepsilon \rightarrow 0$. These two types of scalings for $\mu_{k}^{-}(\varepsilon)-a^{-}$persist depending on the local behavior of $\omega$ in the neighborhood of $j^{-}$. More specifically, 'locally linear' behavior leads to the scaling $\mu_{k}^{-}(\varepsilon)-$ $a^{-} \sim \operatorname{const} \cdot \varepsilon^{\frac{1}{3}}$, while 'locally quadratic' behavior leads to the scaling $\mu_{k}^{-}(\varepsilon)-$ $a^{-} \sim$ const. $\cdot \varepsilon^{\frac{1}{2}}$ as $\varepsilon \rightarrow 0$.

Let us now assume that for $z \approx j^{-}, \omega(z)-a^{-} \sim z-j^{-}$(we will refer to this behavior at the endpoint $z=j^{-}$as 'locally linear'). More precisely, let

$$
\omega(z)=a^{-}+\left(z-j^{-}\right)+\left(z-j^{-}\right)^{2} f(z),
$$

where $f$ is analytic in a neighborhood of $j^{-}$. Then, $\omega$ is univalent in some neighborhood of $j^{-}$; in particular, for $\mu$ sufficiently close to $a^{-}$, we have $\rho:=\mu-a^{-} \sim z_{\mu}-j^{-}$, where $z_{\mu}$ denotes the (simple) turning point as before. Also, $\omega^{-1}(\mu)=j^{-}+\left(\mu-a^{-}\right)+\left(\mu-a^{-}\right)^{2} g(\mu)$, where $g$ is analytic in a neighborhood of $a^{-}$. Now, using (38)

$$
Q^{-}(\mu)=-S\left(j^{-}, \mu\right)=\int_{j^{-}}^{z_{\mu}} \sqrt{i(\omega(\xi)-\mu)} d \xi \sim \operatorname{const.}\left(\omega^{-1}(\mu)-j^{-}\right)^{\frac{3}{2}},
$$


and therefore

$$
Q^{-}(\mu) \sim \text { const. }\left(\left(\mu-a^{-}\right)+g(\mu)\left(\mu-a^{-}\right)^{2}\right)^{\frac{3}{2}} \sim \operatorname{const} .\left(\mu-a^{-}\right)^{\frac{3}{2}} .
$$

More generally, let us assume

$$
\omega(z)=a^{-}+\left(z-j^{-}\right)^{n}+\left(z-j^{-}\right)^{n+1} f(z),
$$

where $f$ is analytic in a neighborhood of $j^{-}$. Firstly, observe that $Q^{-}(\mu)=$ $\left(\mu-a^{-}\right)^{\beta} q^{-}(\mu)$ for some $q^{-}$that is analytic in a neighborhood of $a^{-}$and for some $\beta>0$. For simplicity, let $j^{-}=a^{-}=0$, so that $\omega(z)=z^{n}+z^{n+1} f(z)$. In order to determine $\beta$, we can assume that $\mu$ is real and positive. The transformation $\xi=\mu^{\frac{1}{n}} u$ yields

$$
\begin{aligned}
Q^{-}(\mu) & =\int_{0}^{z_{\mu}} \sqrt{i\left(\xi^{n}+\xi^{n+1} f(\xi)-\mu\right)} d \xi \\
& =e^{-i \pi / 4} \mu^{\frac{1}{2}+\frac{1}{n}} \int_{0}^{u_{\mu}} \sqrt{1-\left(u^{n}+u^{n+1} \mu^{\frac{1}{n}} f\left(\mu^{\frac{1}{n}} u\right)\right)} d u,
\end{aligned}
$$

where $u_{\mu}=\mu^{-\frac{1}{n}} z_{\mu}$. Since $z_{\mu} \sim \mu^{\frac{1}{n}}$ as $\mu \rightarrow 0$, and consequently $u_{\mu} \sim 1$, a straightforward calculus exercise shows that the real integral

$$
\int_{0}^{u_{\mu}} \sqrt{1-\left(u^{n}+u^{n+1} \mu^{\frac{1}{n}} f\left(\mu^{\frac{1}{n}} u\right)\right)} d u \sim \int_{0}^{1} \sqrt{1-u^{n}} d u .
$$

We conclude that $\beta=\frac{n+2}{2 n}$, and hence, $Q^{-}(\mu)=\left(\mu-a^{-}\right)^{\frac{n+2}{2 n}} q^{-}(\mu)$ for some $q^{-}$that is analytic in a neighborhood of $a^{-}$.

We summarize our findings in the following theorem. For the sake of simplicity, in addition to $\bar{a}_{11} \equiv 1$, we assume that $\beta(\zeta) \equiv 1$, so that the spectral problem (39) simply reads

$$
\varepsilon \frac{d^{2} h}{d z^{2}}=i(\omega(z)-\mu) h .
$$

In addition to Assumption 3.3 we make the following assumption on the frequency function $\omega$ :

Assumption 3.10. We assume that

$$
\omega(z)=a^{-}+\left(z-j^{-}\right)^{n^{-}}+\left(z-j^{-}\right)^{n^{-}+1} f^{-}(z)
$$

and

$$
\omega(z)=a^{+}+\left(z-j^{+}\right)^{n^{+}}+\left(z-j^{+}\right)^{n^{+}+1} f^{+}(z)
$$

for some $n^{-}, n^{+} \in \mathbb{N}$ and some functions $f^{-}$and $f^{+}$that analytic in a neighborhood of $j^{-}$and $j^{+}$, respectively. 
Theorem 3.11. Under the Assumptions 3.3 and 3.10, $Q^{-}(\mu)=\left(\mu-a^{-}\right)^{\frac{n^{-}+2}{2 n^{-}}} q^{-}(\mu)$ $\left(Q^{+}(\mu)=\left(\mu-a^{+}\right)^{\frac{n^{+}+2}{2 n^{+}}} q^{+}(\mu)\right)$ for some $q^{-}\left(q^{+}\right)$that is analytic in a neighborhood of $a^{-}\left(a^{+}\right)$. Consequently, $\mu_{k}^{-}(\varepsilon)-a^{-} \sim$ const. $\varepsilon^{\frac{n^{-}}{n^{-}+2}}\left(\mu_{k}^{+}(\varepsilon)-a^{+} \sim\right.$ const. $\left.\varepsilon^{\frac{n^{+}}{n^{+}+2}}\right)$, where the points $\mu_{k}^{ \pm}(\varepsilon)$ are defined in the previous section. Denote by $\alpha_{n}(\varepsilon)=(-i \varepsilon)^{\frac{1}{n+2}}$. Denote by $\left.\mu^{-}(\varepsilon)\left(\mu^{+}\right)(\varepsilon)\right)$ a Dirichlet eigenvalue that lies in the neighborhood of $\gamma^{-}\left(\gamma^{+}\right)$. Then $\mu^{ \pm}(\varepsilon)-a^{ \pm} \sim \rho^{ \pm}\left(\alpha_{n^{ \pm}}(\varepsilon)\right)^{n^{ \pm}}$ as $\varepsilon \rightarrow 0$, where $\rho^{ \pm}$is an eigenvalue of the generalized Airy boundary value problem

$$
\left\{\begin{array}{l}
\frac{d^{2} \chi}{d \zeta^{2}}=\left(\zeta^{n^{ \pm}}-\rho^{ \pm}\right) \chi, \\
\chi(0)=\lim _{r \rightarrow \pm \infty} \chi\left(r \exp \left(\frac{\pi i}{2\left(n^{ \pm}+2\right)}\right)\right)=0 .
\end{array}\right.
$$

Let $\chi$ be a corresponding eigenfunction. Then the eigenfunctions of the problem (48) corresponding to $\mu^{ \pm}(\varepsilon)$ can be obtained in the form

$$
h_{\varepsilon}^{ \pm}(z)=\chi\left(\alpha_{n^{ \pm}}(\varepsilon)^{-1}\left(z-j^{ \pm}\right)\right)[1] .
$$

Proof The proof of the first claim of the theorem follows from the calculations leading up to the statement of the theorem. Left to prove is the last assertion of the theorem. Note that it does not follow from Theorem 3.9 , because the eigenvalue $\mu^{ \pm}(\varepsilon) \rightarrow a^{ \pm}$as $\varepsilon \rightarrow 0$, and the first-order WKB approximation breaks down as $\mu \rightarrow a^{ \pm}$.

Let $\mu=\mu^{-}(\varepsilon)$ be a Dirichlet eigenvalue that lies in the neighborhood of $\gamma^{-}$. We argue by contradiction, i.e., we assume that $\mu^{-}(\varepsilon)-a^{-}=\varepsilon^{\frac{n^{-}}{n^{-}+2}} O(1)$ as $\varepsilon \rightarrow 0$. W.l.o.g., let $j^{-}=a^{-}=0, n=n^{-}$, so that $\omega(z)=z^{n}+z^{n+1} f(z)$, where $f$ is analytic in a neighborhood of 0 . As before, let $z_{\mu}$ denote the (simple) turning point. For the sake of simplicity, let us assume that $f \equiv 0$, i.e., $\omega(z)=z^{n}$. Let $\alpha=(-i \varepsilon)^{\frac{1}{n+2}}$. With the change of variables $\zeta=$ $\alpha^{-1}\left(z-z_{\mu}\right)$, we have

$$
\omega(z)-\mu=\omega(z)-\omega\left(z_{\mu}\right)=\alpha^{n} \sum_{k=0}^{n-1}\left(\begin{array}{l}
n \\
k
\end{array}\right) \zeta^{n-k}\left(\frac{z_{\mu}}{\alpha}\right)^{k}
$$

and the eigenvalue problem (48) with $\zeta_{\mu}^{ \pm}=\alpha^{-1}\left(j^{ \pm}-z_{\mu}\right)$ becomes

$$
\left\{\begin{array}{l}
\frac{d^{2} h}{d \zeta^{2}}=\left(\zeta^{n}+o(1)\right) h \\
h\left(\zeta_{\mu}^{-}\right)=h\left(\zeta_{\mu}^{+}\right)=0
\end{array}\right.
$$


This is a small perturbation of the generalized Airy equation,

$$
\frac{d^{2} h}{d \zeta^{2}}=\zeta^{n} h
$$

Let us define the generalized Airy functions

$$
\begin{aligned}
& A_{n}(\zeta)=p \zeta^{\frac{1}{2}}\left[I_{-p}(\eta)-I_{p}(\eta)\right] \\
& B_{n}(\zeta)=(p \zeta)^{\frac{1}{2}}\left[I_{-p}(\eta)+I_{p}(\eta)\right]
\end{aligned}
$$

where $p=\frac{1}{n+2}, \eta=2 p z^{\frac{1}{2 p}}$ and $I_{p}$ denotes the modified Bessel function of the first kind. From $[14,15]$, we adopt the following:

1. When $n=1$, these $A_{n}$ and $B_{n}$ reduce to the standard Airy functions $\mathrm{Ai}$ and $\mathrm{Bi}$, respectively.

2. They are real on the real axis, analytic in the finite complex plane, and constitute a fundamental set of solutions of the generalized Airy differential equation.

3. Amongst many asymptotic properties, we have

$$
\begin{aligned}
& A_{n}(\zeta)=(p / \pi)^{\frac{1}{2}} \sin (p \pi) \zeta^{-\frac{n}{4}} e^{-\eta}[1+O(1 / \eta)], \quad|\arg (\zeta)|<3 p \pi-\delta \\
& B_{n}(\zeta)=\pi^{-\frac{1}{2}} \zeta^{-\frac{n}{4}} e^{\eta}[1+O(1 / \eta)], \quad|\arg (\zeta)|<p \pi-\delta .
\end{aligned}
$$

4. The zeros of $A_{n}\left(B_{n}\right)$ lie within one of the double-sectors $2 k \pi /(n+1)<$ $|\arg (\zeta)|<(2 k+1) p \pi, 0<2 k<n+1((2 k-1) p \pi<|\arg (\zeta)|<(2 k-$ 1) $\pi /(n+1), 0<2 k<n+3)$, and the zeros within each of these sectors approach one of the rays $|\arg (\zeta)|=(2 k+1) p \pi(|\arg (\zeta)|=(2 k-1) p \pi)$.

The problem (51) has a fundamental set of solutions of the form $h^{-}(\zeta)=$ $A_{n}(\zeta)[1]$ and $h^{+}(\zeta)=B_{n}(\zeta)[1]$, where [1] $=1+o(1)$. As before, the eigenvalues are determined by the equation

$$
\Delta(\mu)=\left|\begin{array}{ll}
h^{+}\left(\zeta_{\mu}^{+}\right) & h^{+}\left(\zeta_{\mu}^{-}\right) \\
h^{-}\left(\zeta_{\mu}^{+}\right) & h^{-}\left(\zeta_{\mu}^{-}\right)
\end{array}\right|=0 .
$$

Without loss of generality, let us rewrite this condition in the form

$$
\frac{A_{n}\left(\zeta_{\mu}^{-}\right)}{B_{n}\left(\zeta_{\mu}^{-}\right)}=\frac{A_{n}\left(\zeta_{\mu}^{+}\right)}{B_{n}\left(\zeta_{\mu}^{+}\right)}[1] .
$$


One can use the asymptotic properties of $A_{n}$ to observe that the righthand side can be majorized by $O(1)\left|\exp \left(\frac{1}{\eta_{\mu}}\right)\right|=O(1) \exp \left(-C \varepsilon^{-\frac{1}{2}}\right)$. By virtue of Rouché's theorem (again we omit technicalities), the above equation has simple roots in the neighborhood of $\mu=\varepsilon^{\frac{n}{n+2}} e^{\frac{i n}{2(n+2)}}(-r)^{n}$ of radius $O(1) \exp \left(-C \varepsilon^{-\frac{1}{2}}\right)$, where $r$ is a zero of $A_{n}$. This is a contradiction to the assumption that $\mu(\varepsilon)=\varepsilon^{\frac{n}{n+2}} O(1)$ as $\varepsilon \rightarrow 0$.

After passing to a subsequence (we omit the subscripts), let us assume that $\lim _{\varepsilon \rightarrow 0} \frac{\mu(\varepsilon)}{\alpha_{n}(\varepsilon)}=\rho$. With the change of variables $\zeta=\alpha^{-1} z$, the spectral problem (48) becomes

$$
\left\{\begin{array}{l}
\frac{d^{2} h}{d \zeta^{2}}=\left(\zeta^{n}+o(1)-\rho\right) h \\
h(0)=h\left(\left(\alpha_{n}(\varepsilon)^{-1} j^{+}\right)=0 .\right.
\end{array}\right.
$$

The conclusion of the theorem follows using the standard arguments.

Lemma 3.12. Let the frequency $\omega$ satisfy the Assumptions 3.3 and 3.10. Let $0<\eta_{1}<\eta=\min \left\{\frac{n^{-}}{n^{-}+2}, \frac{n^{+}}{n^{+}+2}\right\}<\eta_{2}<1$, and let $T_{\varepsilon} \sim$ const. $\varepsilon^{-\eta_{2}}$ as $\varepsilon \rightarrow 0$. Then there exists a constant $M$ independent of $\varepsilon$, so that for any $\stackrel{\circ}{g} \in H_{0}^{1}\left(j^{-}, j^{+}\right)$,

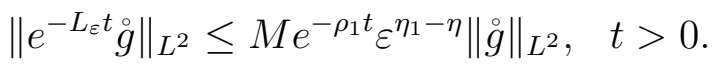

Moreover,

$$
\lim _{\varepsilon \rightarrow 0}\left\|e^{-T_{\varepsilon} L_{\varepsilon}} \stackrel{\circ}{g}\right\|_{H^{1}\left(j^{-}, j^{+}\right)}=0 .
$$

Proof We again, for the sake of clarity assume that $\bar{a}_{11} \equiv 1$ and $\beta(\zeta) \equiv 1$, so that $e^{-L_{\varepsilon} t} g$ represents the solution of the Dirichlet boundary value problem

$$
g_{t}+i \omega g=\varepsilon g_{J J}, \quad g(0)=\stackrel{\circ}{g}, \quad g\left(j^{-}\right)=g\left(j^{+}\right)=0 .
$$

W.l.o.g., let us assume that $\stackrel{\circ}{g} \in C^{\infty}\left(j^{-}, j^{+}\right)$. Note that multiplication by $\bar{g}$ and integration over $\left(j^{-}, j^{+}\right)$leads to

$$
\frac{d}{2 d t}\|g\|_{L^{2}}^{2}+\varepsilon\left\|g_{J}\right\|_{L^{2}}^{2}=0
$$

By virtue of Poincaré inequality, $\left\|g_{J}\right\|_{L^{2}} \geq a^{1 / 2}\|g\|_{L^{2}}, g \in H_{0}^{1}\left(j^{-}, j^{+}\right)$with some constant $a>0$, this leads to the inequality

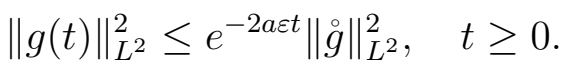


In particular, this implies the dissipation of 'energy' over $T \sim \varepsilon^{-1}$ time scales. In view of relation (34), we can write

$$
e^{-L_{\varepsilon} t \stackrel{\circ}{g}}=e^{-\lambda_{1} t} \sum_{r=1}^{\infty} \sum_{n=N(r)+1}^{N(r+1)} e^{-\left(\lambda_{n}-\lambda_{1}\right) t}\left\langle\stackrel{\circ}{g}, \bar{g}_{n}\right\rangle g_{n}=: e^{-\lambda_{1} t} u(t),
$$

and, therefore $\left\|e^{-L_{\varepsilon} t} \stackrel{\circ}{g}\right\|_{L^{2}}=e^{-\rho_{1} t}\|u(t)\|_{L^{2}}$. It is obvious that $\lim _{t \rightarrow 0^{+}} u(t)=\stackrel{\circ}{g}$ and $\lim _{t \rightarrow \infty} u(t)=0$, with both limits understood in the $L^{2}$-metric. Let $\delta_{\varepsilon} \sim \varepsilon^{\eta_{1}}$ be such that the knot $\mu_{0} \notin U_{\delta_{\varepsilon}}\left(a^{-}\right) \cup U_{\delta_{\varepsilon}}\left(a^{+}\right)$, and let $N_{\varepsilon} \in \mathbb{N}$ be such that $\lambda_{n} \in U_{\delta_{\varepsilon}}\left(i a^{-}\right) \cup U_{\delta_{\varepsilon}}\left(i a^{+}\right)$if and only if $n \leq N_{\varepsilon}$. From the previous theorem, we infer easily that $N_{\varepsilon} \sim \varepsilon^{\eta_{1}-\eta}$ as $\varepsilon \rightarrow 0$. W.l.o.g., let us assume that there exists $r_{\varepsilon}$ such that $N\left(r_{\varepsilon}\right)=N_{\varepsilon}$. Then we have

$$
\|u(t)\|_{L^{2}} \leq\|g\|_{L^{2}} \sum_{n=1}^{N_{\varepsilon}} \kappa_{n}+\sum_{r=r_{\varepsilon}}^{\infty} \sum_{n=N(r)+1}^{N(r+1)} e^{-\left(\lambda_{n}-\lambda_{1}\right) t}\left\langle\stackrel{\circ}{g}, \bar{g}_{n}\right\rangle g_{n} .
$$

By virtue of the normalization and the approximation (50) of the eigenfunctions, the condition numbers $\kappa_{n}, n \leq N_{\varepsilon}$ are bounded independently of $\varepsilon$. Due to $\lambda_{1}=o\left(\lambda_{n}\right)$ for $n \geq N_{\varepsilon}$ as $\varepsilon \rightarrow 0$, and the stability of the Abel-Lidskii summation formula, we infer that there exists a constant $M$ so that (53) holds.

Taking a derivative with respect with $J$ in the PDE above, and denoting $G=g_{J}$ we obtain

$$
G_{t}+i \omega G+i \omega^{\prime} g=\varepsilon G_{J J}, \quad G(0)=\stackrel{\circ}{g}_{J}
$$

This is a non-homogeneous equation in $G$, and it satisfies non-homogeneous Dirichlet boundary conditions as well. We deal with this difficulty by noticing that the entire analysis we devised for the homogeneous Dirichlet boundary value problem $L_{\varepsilon} g=\lambda g$ carries over for the non-homogeneous Dirichlet boundary value problem as well. The solution of the above equation is basically the solution of the same equation with homogeneous boundary value problem modulo projection on a single mode satisfying the non-homogeneous Dirichlet boundary value problem $L_{\varepsilon} g=\lambda g$. Therefore, w.l.o.g., we can assume homogeneous boundary value condition. For the sake of clarity, let us additionally assume that $1=\sup _{J \in\left[j^{-}, j^{+}\right]} \omega^{\prime}(J)$. Multiplying the equation by $G$ and integrating by parts leads similarly as before to

$$
\frac{d}{2 d t}\|g\|_{L^{2}}^{2}+\varepsilon\left\|g_{J}\right\|_{L^{2}}^{2} \leq \frac{\|g\|_{L^{2}}^{2}}{2 a \varepsilon}+\frac{a \varepsilon}{2}\|g\|_{L^{2}}^{2}
$$


and hence

$$
\frac{d}{d t}\|g\|_{L^{2}}^{2}+a \varepsilon\|g\|_{L^{2}}^{2} \leq \frac{\|g\|_{L^{2}}^{2}}{a \varepsilon}
$$

Finally, for $t^{\prime} \leq t$ we have

$$
\begin{aligned}
\|g(t)\|_{L^{2}}^{2} & \leq\left\|g\left(t^{\prime}\right)\right\|_{L^{2}}^{2} e^{-a \varepsilon\left(t-t^{\prime}\right)}+\int_{t^{\prime}}^{t} \frac{\|g(\tau)\|_{L^{2}}^{2}}{a \varepsilon} e^{a \varepsilon(\tau-t)} d \tau \\
& \leq e^{-a \varepsilon\left(t-t^{\prime}\right)}\left(\left\|g\left(t^{\prime}\right)\right\|_{L^{2}}^{2}+\frac{\left\|g\left(t^{\prime}\right)\right\|_{L^{2}}^{2}}{a^{2} \varepsilon^{2}}\right) .
\end{aligned}
$$

In particular, $\left\|g\left(T_{\varepsilon}\right)\right\|_{L^{2}}^{2} \leq e^{-a \varepsilon T_{\varepsilon}}\left(\|g(0)\|_{L^{2}}^{2}+\frac{\|g(0)\|_{L^{2}}^{2}}{a^{2} \varepsilon^{2}}\right)$. For $t \geq T_{\varepsilon}$, we can write $G(t)=G^{1}(t)+G^{2}(t)$, where

$$
G_{t}^{1}+i \omega G^{1}+i \omega^{\prime} g=\varepsilon G_{J J}^{1}, \quad G^{1}\left(T_{\varepsilon}\right)=0
$$

and

$$
G_{t}^{2}+i \omega G^{2}=\varepsilon G_{J J}^{2}, \quad G^{2}\left(T_{\varepsilon}\right)=G\left(T_{\varepsilon}\right) .
$$

Note that similarly as above, we have

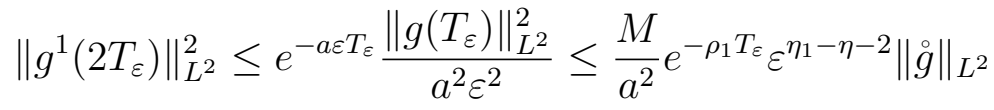

and

$$
\left\|g^{2}\left(2 T_{\varepsilon}\right)\right\|_{L^{2}}^{2} \leq M e^{-\rho_{1} T_{\varepsilon}} \varepsilon^{\eta_{1}-\eta}\left\|g\left(T_{\varepsilon}\right)\right\|_{L^{2}} \leq e^{-\rho_{1} T_{\varepsilon}} M \varepsilon^{\eta_{1}-\eta}\left(\|g(0)\|_{L^{2}}^{2}+\frac{\|g(0)\|_{L^{2}}^{2}}{a^{2} \varepsilon^{2}}\right) .
$$

The second assertion of the theorem follows immediately.

Theorem 3.13. Under the same assumptions of the previous lemma, for any initial datum $\stackrel{v}{\in} H_{0}^{1}(D)$, we have

$$
\lim _{\varepsilon \rightarrow 0}\left\|c^{(\varepsilon)}\left(T_{\varepsilon}\right)-\bar{v}\left(\varepsilon T_{\varepsilon}\right)\right\|_{L^{2}(D)}=0
$$

where $c^{(\varepsilon)}$ and $\bar{v}$ are the solutions of equation (4) and equation (9) with ini-

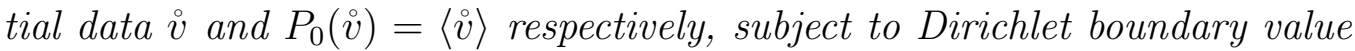
conditions. Furthermore, the $L^{2}$-norm in (54) can be replaced by the stronger $L^{\infty}$-norm. 
Proof Let $\stackrel{v}{=} \stackrel{\circ}{v}_{D}+\stackrel{\circ}{v}_{C} \in H_{D} \oplus H_{C}=H_{0}^{1}(D)$. W.l.o.g., we can assume $\stackrel{v}{v} \in C^{\infty}(D)$ and $\dot{v}_{D}=P_{0} \stackrel{\circ}{v}$ and $\dot{v}_{C}=P_{1} \stackrel{\circ}{v}$, with projections $P_{0}$ and $P_{1}$ defined in (15). The solution $v^{(\varepsilon)}$ to the initial datum $\dot{v}$ of Dirichlet boundary value problem (7) can now be written as

$$
v^{(\varepsilon)}(t)=v_{D}^{(\varepsilon)}+v_{C}^{(\varepsilon)}(t)=\bar{v}(\varepsilon t)+e^{-L_{\varepsilon} t} \dot{v}_{C} \quad t>0,
$$

where $\bar{v}$ is the solution of the effective diffusive equation (9) with $\bar{v}(0)=\dot{v}_{D}$. By the previous lemma, it follows immediately that as $\varepsilon \rightarrow 0$,

$$
\left\|v^{(\varepsilon)}\left(T_{\varepsilon}\right)-\bar{v}\left(\varepsilon T_{\varepsilon}\right)\right\|_{L^{2}}=\left\|e^{-L_{\varepsilon} T_{\varepsilon}} \dot{o}_{C}\right\|_{L^{2}} \rightarrow 0 .
$$

Let now $c^{(\varepsilon)}$ be the solution to the initial datum $\stackrel{\circ}{v}=\stackrel{\circ}{v}_{D}+\dot{\circ}_{C}$ of the Dirichlet boundary value problem (4). We again make the ansatz $c^{(\varepsilon)}=v^{(\varepsilon)}+\varepsilon \mathfrak{u}^{(\varepsilon)}$, where $v^{(\varepsilon)}$ and $\mathfrak{u}^{(\varepsilon)}$ solve Dirichlet boundary value problems (7) and (8) with $v^{(\varepsilon)}(0)=\stackrel{v}{v}$ and $\mathfrak{u}^{(\varepsilon)}(0)=0$, respectively. We need to prove that $\left\|\mathfrak{u}_{\varepsilon}\left(T_{\varepsilon}\right)\right\|_{L^{2}}$ is bounded independently of $\varepsilon$. We write $\mathfrak{u}^{(\varepsilon)}=\mathfrak{u}_{D}^{(\varepsilon)}+\mathfrak{u}_{C}^{(\varepsilon)}$, where $\mathfrak{u}_{D}^{(\varepsilon)}$ and $\mathfrak{u}_{C}^{(\varepsilon)}$ respectively satisfy

$$
\mathfrak{u}_{t}+\omega(J) \mathfrak{u}_{\theta}=\varepsilon \Delta_{(J, \theta)} \mathfrak{u}+\mathcal{B}(J, \theta) v_{D}^{(\varepsilon)},
$$

and

$$
\mathfrak{u}_{t}+\omega(J) \mathfrak{u}_{\theta}=\varepsilon \Delta_{(J, \theta)} \mathfrak{u}+\mathcal{B}(J, \theta) v_{C}^{(\varepsilon)},
$$

both with $\mathfrak{u}_{D}^{(\varepsilon)}(0)=\mathfrak{u}_{C}^{(\varepsilon)}(0)=0$. Since $\left\langle\mathcal{B} v_{D}\right\rangle=0$, the equation $\omega(J) \mathfrak{v}_{\theta}=$ $\mathcal{B}(J, \theta) v_{D}$ possesses a first integral $\mathfrak{v} \in H_{0}^{1}$. Consequently, $w:=\mathfrak{u}_{D}^{(\varepsilon)}-\mathfrak{v}$ satisfies

$$
w_{t}+\omega(J) w_{\theta}=\varepsilon \Delta_{(J, \theta)} w+\varepsilon \Delta_{(J, \theta)} \mathfrak{v} .
$$

Scalar multiplication by $w$ in $L^{2}$ together with the Poincaré inequality (denoting the constant again by $a$ )

$$
\left\|\nabla_{(J, \theta)} w\right\|_{L^{2}}^{2} \geq a\|w\|_{L^{2}}^{2}, w \in H_{0}^{1}(D),
$$

yield

$$
\frac{d}{d t}\|w\|_{L^{2}}^{2}+a \varepsilon\|w\|_{L^{2}}^{2} \leq \frac{\varepsilon}{a}\left\|\Delta_{(J, \theta)} \mathfrak{v}\right\|_{L^{2}}^{2} .
$$

It easily follows that $\left\|\mathfrak{u}_{D}^{(\varepsilon)}\right\|_{L^{2}}$ is bounded independently of $\varepsilon$.

To show the same for $\mathfrak{u}_{C}^{(\varepsilon)}$, note that it satisfies two equations,

$$
\mathfrak{u}_{t}+\mathcal{L}_{\varepsilon} \mathfrak{u}=\mathcal{B}(J, \theta) v_{C}^{(\varepsilon)}=\frac{1}{\varepsilon}\left(\left\langle\mathcal{L}_{\varepsilon}\right\rangle-\mathcal{L}_{\varepsilon}\right) v_{C}^{(\varepsilon)},
$$


and

$$
\mathfrak{u}_{t}+\left\langle\mathcal{L}_{\varepsilon}\right\rangle \mathfrak{u}=\mathcal{B}(J, \theta) c_{C}^{(\varepsilon)}=\frac{1}{\varepsilon}\left(\left\langle\mathcal{L}_{\varepsilon}\right\rangle-\mathcal{L}_{\varepsilon}\right) c_{C}^{(\varepsilon)} .
$$

Recall that $P_{0} \mathfrak{u}_{C}^{(\varepsilon)}=0$, hence that after taking the projection $P_{1}$, and recalling the notation $L_{\varepsilon}=\left\langle\mathcal{L}_{1, \varepsilon}\right\rangle$, the second equation is equivalent to

$$
\mathfrak{u}_{t}+L_{\varepsilon} \mathfrak{u}=P_{1} \mathcal{B} c^{(\varepsilon)}=\frac{1}{\varepsilon} P_{1}\left(\left(\left\langle\mathcal{L}_{\varepsilon}\right\rangle-\mathcal{L}_{\varepsilon}\right) c^{(\varepsilon)}\right) .
$$

Note that $f=\frac{1}{\varepsilon} P_{1}\left(\left(\left\langle\mathcal{L}_{\varepsilon}\right\rangle-\mathcal{L}_{\varepsilon}\right) v_{C}^{(\varepsilon)}\right)$ satisfies

$$
f_{t}+\left\langle\mathcal{L}_{\varepsilon}\right\rangle f=\frac{1}{\varepsilon} P_{1}\left(\left[\mathcal{L}_{\varepsilon},\left\langle\mathcal{L}_{\varepsilon}\right\rangle\right] v_{C}^{(\varepsilon)}\right),
$$

where $\left[\mathcal{L}_{\varepsilon},\left\langle\mathcal{L}_{\varepsilon}\right\rangle\right]=\mathcal{L}_{\varepsilon}\left\langle\mathcal{L}_{\varepsilon}\right\rangle-\left\langle\mathcal{L}_{\varepsilon}\right\rangle \mathcal{L}_{\varepsilon}$ denotes the commutator, a differential operator of degree one. Therefore,

$$
f_{t}+L_{\varepsilon} f=\frac{1}{\varepsilon} P_{1}\left[\mathcal{L}_{\varepsilon},\left\langle\mathcal{L}_{\varepsilon}\right\rangle\right] v_{C}^{(\varepsilon)} .
$$

Similarly as in the the proof of the last lemma, one can now prove that there exists a constant $C$ not depending on $\varepsilon$ and a constant $k>0$ such that

$$
\|f(t)\|_{L^{2}}^{2} \leq e^{-\rho_{1} T_{\varepsilon}} C \varepsilon^{-k}, \quad t \in\left[T_{\varepsilon}, 2 T_{\varepsilon}\right] .
$$

We write $\mathfrak{u}(t)=\mathfrak{u}^{1}(t)+\mathfrak{u}^{2}(t), t \in\left[T_{\varepsilon}, 2 T_{\varepsilon}\right]$, where

$$
\mathfrak{u}_{t}^{1}+\mathcal{L}_{\varepsilon} \mathfrak{u}^{1}=f, \quad \mathfrak{u}^{1}\left(T_{\varepsilon}\right)=0
$$

and

$$
\mathfrak{u}_{t}^{2}+\left\langle\mathcal{L}_{\varepsilon}\right\rangle \mathfrak{u}^{2}=0, \quad \mathfrak{u}^{2}\left(T_{\varepsilon}\right)=\mathfrak{u}\left(T_{\varepsilon}\right) .
$$

Similarly as in the proof for the last lemma, we obtain

$$
\begin{aligned}
\left\|\mathfrak{u}^{1}\left(2 T_{\varepsilon}\right)\right\|_{L^{2}}^{2} & \leq\left\|\mathfrak{u}^{1}\left(T_{\varepsilon}\right)\right\|_{L^{2}}^{2} e^{-a \varepsilon T_{\varepsilon}}+\int_{T_{\varepsilon}}^{2 T_{\varepsilon}} \frac{\|f(\tau)\|_{L^{2}}^{2}}{a \varepsilon} e^{a \varepsilon(\tau-t)} d \tau \\
& \leq \frac{C}{a^{2}} e^{-\rho_{1} T_{\varepsilon}} \varepsilon^{-k-2} .
\end{aligned}
$$

On the other hand,

$$
\left\|\mathfrak{u}^{2}\left(2 T_{\varepsilon}\right)\right\|_{L^{2}} \leq e^{-\rho_{1} T_{\varepsilon}} M \varepsilon^{\eta_{1}-\eta}\left\|\mathfrak{u}\left(T_{\varepsilon}\right)\right\|_{L^{2}} .
$$


We conclude that $\left\|\mathfrak{u}\left(2 T_{\varepsilon}\right)\right\|_{L^{2}}$ is bounded independently of $\varepsilon$, and the conclusion follows from (55). For the fact that $L^{2}$ norm can be replaced by $L^{\infty}$ norm we refer the reader to the following estimate that can be found in Lemma 5.4. in [35]:

$$
\|c(t)\|_{L^{\infty}} \leq C t^{-\frac{1}{2}}\|c(0)\|_{L^{2}}, \quad t \in(0,1] .
$$

This completes the proof.

\subsubsection{Radial flows with monotonic frequency}

We apply the previous result to some important examples.

(a) In the case of a (regularized) vortical flow, $\omega(r)=\frac{1}{b^{2}+r^{2}}, b \geq 0$, observe that $\omega$ is locally quadratic in the neighborhood of $r^{-}=0$ and locally linear in the neighborhood of $r^{-}>0$. It follows that when the domain is a disk $\left(r^{-}=0\right)$, we have $\mu(\varepsilon)-a^{-} \sim$ const. $\varepsilon^{\frac{1}{2}}$, and when the domain is an annulus $\left(r^{-}>0\right), \mu(\varepsilon)-a^{-} \sim$ const. $\varepsilon^{\frac{1}{3}}$, where $\mu(\varepsilon)$ are the eigenvalues near $\gamma^{-}$. For the branch near $\gamma^{+}, \omega$ it is locally linear in either case leading to $\mu(\varepsilon)-a^{+} \sim$ const. $\varepsilon^{\frac{1}{3}}$.

(b) The situation is similar in the case of the two-dimensional creeping Couette flow between two concentric cylinders of radii $0<r^{-}<r^{+}$with the outer cylinder moving with velocity $\Omega r^{+}$. The velocity field is given by

$$
v_{\theta}(r)=\Omega r \frac{1-\left(r^{-} / r\right)^{2}}{1-\left(r^{-} / r^{+}\right)^{2}}
$$

so that the potential $\omega(r)=v_{\theta}(r) / r$ is increasing and it is locally linear at both $r^{ \pm}$leading to $\mu(\varepsilon)-a^{ \pm} \sim$ const. $\varepsilon^{\frac{1}{3}}$ on both branches.

\subsection{Parabolic frequency}

The above developed theory becomes much more involved if $\omega$ is not monotonic. However, the problem is still tractable if $\omega:\left[j^{-}, j^{+}\right] \rightarrow \mathbb{R}$ is such that it decreases on $\left[j^{-}, j^{c}\right]$ and increases on $\left[j^{c}, j^{+}\right]$(see [31]). Assume for simplicity that $a^{c}=\omega\left(j^{c}\right)<a^{-}=\omega\left(j^{-}\right)<a^{+}=\omega\left(j^{+}\right)$, and let $\Pi^{ \pm}=$ $\left\{\mu \mid \Im(\mu)<0, \Re(\mu) \in\left(a^{c}, a^{ \pm}\right)\right\}$. Let $G^{ \pm}$be the preimage of $\Pi^{ \pm}$under $\omega$ as before. The equation $\omega(j)=\mu$ has two unique roots $j_{\mu}^{ \pm} \in G^{ \pm}$. Similarly as before, we define the functions

$$
Q_{-}^{ \pm}(\mu)= \pm \int_{j_{\mu}^{-}}^{j^{ \pm}} \sqrt{i(\omega(\xi)-\mu)} d \xi \text { and } Q_{+}^{ \pm}(\mu)= \pm \int_{j_{\mu}^{+}}^{j^{ \pm}} \sqrt{i(\omega(\xi)-\mu)} d \xi
$$


The four level sets,

$$
\tilde{\gamma}_{ \pm}^{ \pm}=\left\{\mu \in \overline{\Pi^{ \pm}} \mid \Re Q_{ \pm}^{ \pm}(\mu)=0\right\}
$$

are referred to as singular lines. In addition, we define

$$
Q^{c}(\mu)=\int_{j_{\mu}^{-}}^{j_{\mu}^{+}} \sqrt{i(\omega(\xi)-\mu)} d \xi \text { and } Q^{\infty}(\mu)=\int_{j^{-}}^{j^{+}} \sqrt{i(\omega(\xi)-\mu)} d \xi
$$

We now define the principal line

$$
\tilde{\gamma}_{\infty}=\left\{\mu \in \overline{\Pi^{+}} \mid \Re Q(\mu)=0\right\}
$$

and the critical line

$$
\tilde{\gamma}_{c}=\left\{\mu \in \overline{\Pi^{+}} \mid \Re Q^{c}(\mu)=0\right\} .
$$

Let $\tilde{\Gamma}$ be the union of these six lines. The spectral limit graph $\Gamma$ has a much more complicated structure (it is a subset of $\tilde{\Gamma}$ ), and it is beyond the scope of this paper to describe the whole structure (for more details, the reader is referred to [31]). For the purposes of this paper, the important observation is that there are three lines of $\Gamma$ emerging from $a^{c}, a^{-}$and $a^{+}: \gamma_{c}, \gamma_{-}^{-}$and $\gamma_{+}^{+}$, respectively. The scaling of eigenvalues with respect to $\varepsilon$ along these lines can be determined in a similar fashion as before from the local behavior of $\omega(j)$ in the neighborhood of $j^{c}, j^{-}$and $j^{+}$, respectively.

The above situation applies for example in the case of the parabolic MHDdriven Poiseuille profile $v(r)=C\left(r-r^{-}\right)\left(r-r^{+}\right)$. In this case $\omega(r)=$ $C\left(r-r^{-}\right)\left(r^{+}-r\right) / r$ and the above set-up applies with $r^{c}=\sqrt{r^{-} r^{+}}$. A similar analysis as before shows that the locally quadratic behavior at $r^{c}$ leads to the scaling $\mu(\varepsilon)-a^{c} \sim$ const. $\varepsilon^{\frac{1}{2}}$, and the locally linear behavior at $r^{ \pm}$leads to the scaling $\mu(\varepsilon)-a^{ \pm} \sim$ const. $\varepsilon^{\frac{1}{3}}$.

\section{Numerical comparisons}

We illustrate the results by numerically computing the spectrum for the Poiseuille profile with $r^{-}=0.25$ and $r^{+}=1$. using a standard Chebyshev polynomial $(N=84)$ co-location scheme and ARPACK to solve the resulting eigenvalue problem (32). The spectral graph (Fig. 3) for $m=1$ shows the two main solution branches localizing on the right end point and the critical 
point at $r=1 / 2$ as shown in the left panel of Fig. 4. The right panel of Fig. 4 clearly indicates the expected scaling with $\epsilon$ for the two branches.

Let us now compare numerical solutions of the original equation (29) and the averaged equation to the first order (30). We consider the evolution of the tracer field on a unit disk $(0 \leq r \leq 1,0 \leq \theta \leq 2 \pi)$ with zero Dirichlet boundary conditions $v(r=1, \theta)=0$. We compare two solutions of the equations (29) and (30) at Poincaré sections where $F=0$. We use Chebychev spectral methods to numerically approximate spatial differentiation operators and a second order Crank-Nicolson finite difference scheme in time. For numerics the following parameters were chosen: $a=0.05, f(t)=\sin (2 \pi t / T)$ and $T=1$. For this choice of the advective force parameters $\langle F\rangle$ and $\left\langle F^{2}\right\rangle$ are found to be $1 / 2 \pi$ and $3 / 8 \pi^{2}$ correspondingly. Fig 5 represents 10 periods of evolution for some initial state, which is taken to be $v_{0}(r, \theta)=r e^{-b r^{2}} \cos (\pi r / 2)$.

In order to get a better understanding of the differences between the behavior of the full and approximate equations we introduce the following operators (for both the exact and averaged equations) that maps the scalar field between two associated Poincaré sections:

$$
\begin{aligned}
\mathcal{Q}: & u(r, \theta, t+T) & =\mathcal{Q} u(r, \theta, t), \\
\mathcal{Q}_{\mathrm{av}}: & u_{\mathrm{av}}(r, \theta, t+T) & =\mathcal{Q}_{\mathrm{av}} u_{\mathrm{av}}(r, \theta, t)
\end{aligned}
$$

We can now study how the eigenvalues $\mathcal{Q} \psi_{j}=\lambda_{j} \psi_{j}$ of the above operators change with $\varepsilon$. To do that we introduce relative difference in the eigenvalues of operators as

$$
\delta \lambda_{j}=\frac{\left|\lambda_{j}^{(\mathrm{full})}-\lambda_{j}^{(\mathrm{av})}\right|}{\lambda_{j}^{(\mathrm{full})}}
$$

Here $\lambda_{j}^{(\text {full })}$ and $\lambda_{j}^{(\text {av) }}$ are $j$-th eigenvalues of the full and averaged operator. In Fig. 6 we present $\delta \lambda_{j}(\varepsilon)$ for several eigenmodes.

It is immediately seen from this plot that modes 1 and 13 are almost identical for any value of the diffusion. This follows from the observation that these modes possess axial symmetry, and, therefore, the procedure of averaging does not have any effect on the one-period evolution. For the modes that possess axial symmetry advection-diffusion equation (1) reduces to heat equation since advection implies only rotational translation. We also conclude that $\delta \lambda_{j} \sim \varepsilon^{\alpha}$ where $\alpha$ is found to be equal $\alpha=0.82$.

We now consider flow with time dependence in the form

$$
f(t)=f_{0}(t)+f_{1},
$$


where $f_{0}(t)$ is periodic and mean-free function of time and $f_{1}$ is a constant. The motion corresponds to the rotation of the system as a whole with a constant angular velocity $\omega$ (which still is a function of $r$ ) and periodic oscillations superposed with this rotational motion. Because of the fact that rotational motion is dependent upon $r$, large gradients are constantly created in the scalar field. These gradients are exposed to the action of diffusive smearing. The enhanced stretching of the tracer field creates somewhat richer dynamics and provides for faster mixing. We demonstrate evolution of the initial state for the case of the flow (58) and $\varepsilon=0.01$ in the Fig. 7. Clearly, states as computed using full time-dependent operator and averaged operator are almost indistinguishable.

We finally show evolution of the system for the case of very small effective diffusivity $\varepsilon=10^{-5}$. For such small value of the parameter $\varepsilon$, tracer field does not diffuse trough the boundary for a long time, and, therefore, large twists can be created by the mean component of the circular flow. We illustrate it in Fig. 9.

\section{Conclusion}

We consider the two-dimensional advection-diffusion equation. For Liouville integrable Hamiltonians, we derive an averaged equation and study the corresponding boundary value spectral problem. Separation of variables leads to a self-adjoint effective diffusion equation and a countable family of nonself-adjoint 1D Schrd̈inger equations. For the latter, we study the spectra using complex-plane WKBJ methods. We establish sublinear power scaling laws with respect to the diffusivity, and infer from them enhanced dissipation timescales. Numerical comparison of the spectra of the full equation and the averaged equation shows convergence of the spectra in the limit of vanishing diffusivity.

\section{Acknowledgments}

This work was partially supported by the following NSF grants: DMS1009461, DMS-0807396, DMS-1108780, and CNS-0855217 and the Office of Naval Research MURI OCEAN 3D + 1 grant N00014-11-1-0087. The authors also acknowledge support from the Initiative for the Theoretical Sciences (ITS) at the CUNY Graduate Center. 


\section{References}

[1] S. Agmon. Lectures on Elliptic Boundary Value Problems. Van Nostrand, Princeton, 1965.

[2] M. Faierman. On the spectral theory of an elliptic boundary value problem involving an indefinite weight. Operator Theory and Boundary Eigenvalue Problems. Edited by I. Gohberg and H. Langer, Birkhuser Verlag, Basel, 1995: 137-154.

[3] Batchelor G. K. Small-scale variations of convected quantities like temperature in turbulent fluid. Journal of Fluid Mechanics., 5:113-133, 1959.

[4] H. Berestycki, F. Hamel and N. Nadirashvili. Elliptic eigenvalue problems with large drift and applications to nonlinear propagation phenomena. Comm. Math. Phys., 253:451-480, 2005.

[5] P. Constantin, A. Kiselev, L. Ryzhik, and A. Zlatoš. Diffusion and Mixing in Fluid Flow. Annals of Math. 168:643-674, 2008.

[6] E. B. Davies. Linear Operators and Their Spectra Cambridge University Press, New York, 2007.

[7] E. B. Davies, A. B. J. Kuijlaars Spectral asymptotic of the non-selfadjoint harmonic oscillator. J. London Math. Soc. (2) 70:420-426, 2004.

[8] E. B. Davies. Wild spectral behaviour of anharmonic oscillators. Bull. London Math. Soc. 32:432-438, 2000.

[9] Y. V. Kurylev and M. Lassas. Abel-Lidskii basis in the non-self-adjoint inverse boundary problem. J. Math. Sci 102(4):4237-4257, 2000.

[10] M. S. Agranovich. Elliptic boundary problems newblock Encyclopedia of Mathematical Sciences, Springer Verlag Berlin Heidelberg, 1997.

[11] M. S. Agranovich. Elliptic operators on closed manifolds. Encyclopedia of Mathematical Sciences, Springer Verlag Berlin Heidelberg, 1994.

[12] A. Fannjiang and G. Papanicolau. Convection enhanced diffusion for periodic flows. SIAM Jour. Appl. Math., 54:333-408, 1994 
[13] M. V. Fedoryuk Asymptotic analysis: Linear ordinary differential equations. Springer Verlag, Berlin Heidelberg, 1993

[14] C. A. Swanson and V. K. Barwell. On the distribution of the zeros of the generalized Airy functions. Math. Comp., 29(131):863-877, 1975.

[15] V. B. Headley. An extension of Airy's equation. SIAM Jour. Appl. Math., 15(6):1400-1412, 1967.

[16] G. A. Pavliotis. Homoenization theory for advection-diffusion equation with the mean flow. PhD thesis, Rensselaer Polytechnic Institute, Troy, New York, 2002.

[17] R. M. McLaughlin J. Bonn. Sensitive enhanced diffusivities for flows with fluctuating mean winds: A two-parameter study. Journal of Fluid Mechanics., 445:345 - 375, 2001.

[18] A.H. Nayfeh. Perturbation methods. Wiley International, 1973.

[19] M. Freidlin and A. Wentzell. Random Perturbations of Dynamical Systems. Springer-Verlag, Berlin Heidelberg, 3rd edition, 2012.

[20] M. Freidlin and A. Wentzell. Random perturbations of Hamiltonian systems. Memoir AMS 109 523, 1994.

[21] M. Freidlin and A. Wentzell. Diffusion Processes on Graphs and the Averaging Principle. Ann. Prob. 21:2215-2245, 1993.

[22] M. Freidlin and A. Wentzell. Averaging principle for quasi-linear parabolic PDEs and related diffusion processes. Stochastics and Dynamics, 12 (01): 1150008, 20012.

[23] M. Giona, S. Cerbelli, and V. Vitacolonna. Universality and imaginary potentials in advectiondiffusion equations in closed flows. J. Fluid Mech., 513: 221-237, 2004.

[24] M. Giona, V. Vitacolonna, S. Cerbelli, and A. Adrover. Advection diffusion in nonchaotic closed flows: Non-Hermitian operators, universality, and localization. Phys. Rev. E, 70:046224, 1-12, 2004.

[25] J. P. Gleeson. Transient micromixing: examples of laminar and chaotic stirring. Physics of Fluids, 17:100614, 2005. 
[26] J. P. Gleeson, J. West, O. M. Roche, and A. Gelb. Modelling annular micromixers. SIAM J. Appl. Math., 64(4), 1294-1310, 2004.

[27] L. Koralov . Random Perturbations of 2-dimensional Hamiltonian Flows. Probab. Theory and Related Fields, 129: 37-62, 2004.

[28] S. K. Turitsyn I. Gabitov, T. Schäfer. Lie-transform averaging in nonlinear optical transmission systems with strong and rapid periodic dispersion variations. Phys. Lett. A, 265:274-281, 2000.

[29] S. N. Tumanov and A. A. Shkalikov. On the limit behaviour of the spectrum of a model problem for the OrrSommerfeld equation with Poiseuille profile. Izv. RAN. Ser. Mat., 66 (4):177-204, 2002

[30] A. A. Shkalikov. Spectral Portraits of the OrrSommerfeld Operator with Large Reynolds Numbers. J. Math. Sci., 124 (6): 5417-5441, 2004.

[31] V. I. Pokotilo and A. A. Shkalikov. Semiclassical Approximation for a Nonself-Adjoint SturmLiouville Problem with a Parabolic Potential. Mat. Zametki, 86, (3): 469-473, 2009.

[32] Tobias Schäfer, Andrew C. Poje, and Jesenko Vukadinovic. Averaged dynamics of time-periodic advection diffusion equations in the limit of small diffusivity. Physica D: Nonlinear Phenomena, 238:233-240, 2009.

[33] Frank M. White. Fluid Mechanics. McGraw Hill, 4th edition, 1999.

[34] V. I. Arnold. Mathematical Methods of Classical Mechanics. Springer, New York, 1989.

[35] A. Zlatoš. Diffusion in fluid flow: Dissipation enhancement by flows in 2D. Comm. Partial Differential Equations, 35:496-534, 2010. 


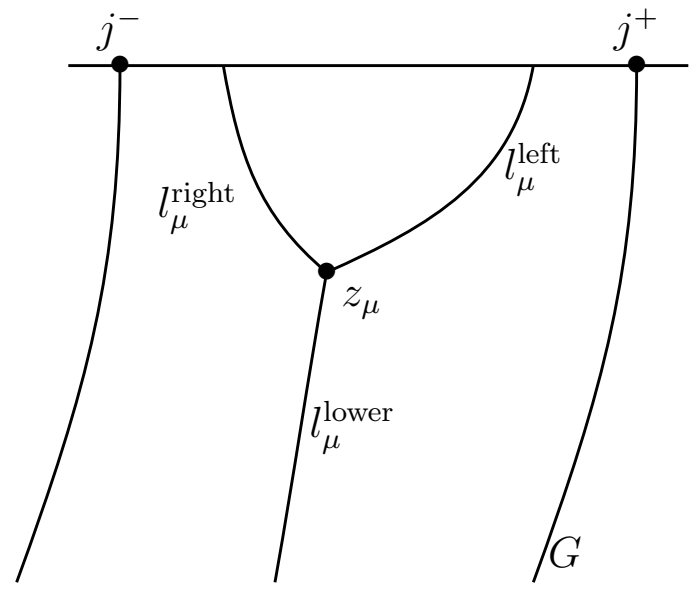

Figure 1: Sketch of geometry and notation as given in Assumption 3.3

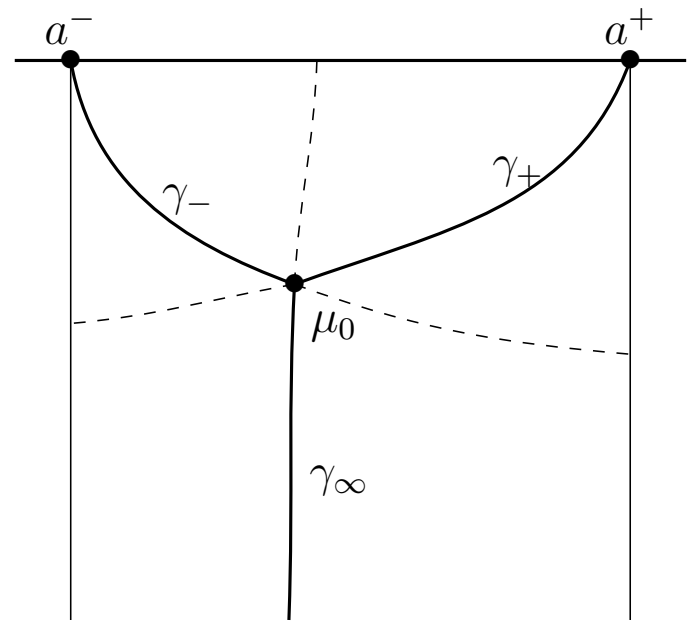

Figure 2: Geometry and notation of Theorem 3.6 


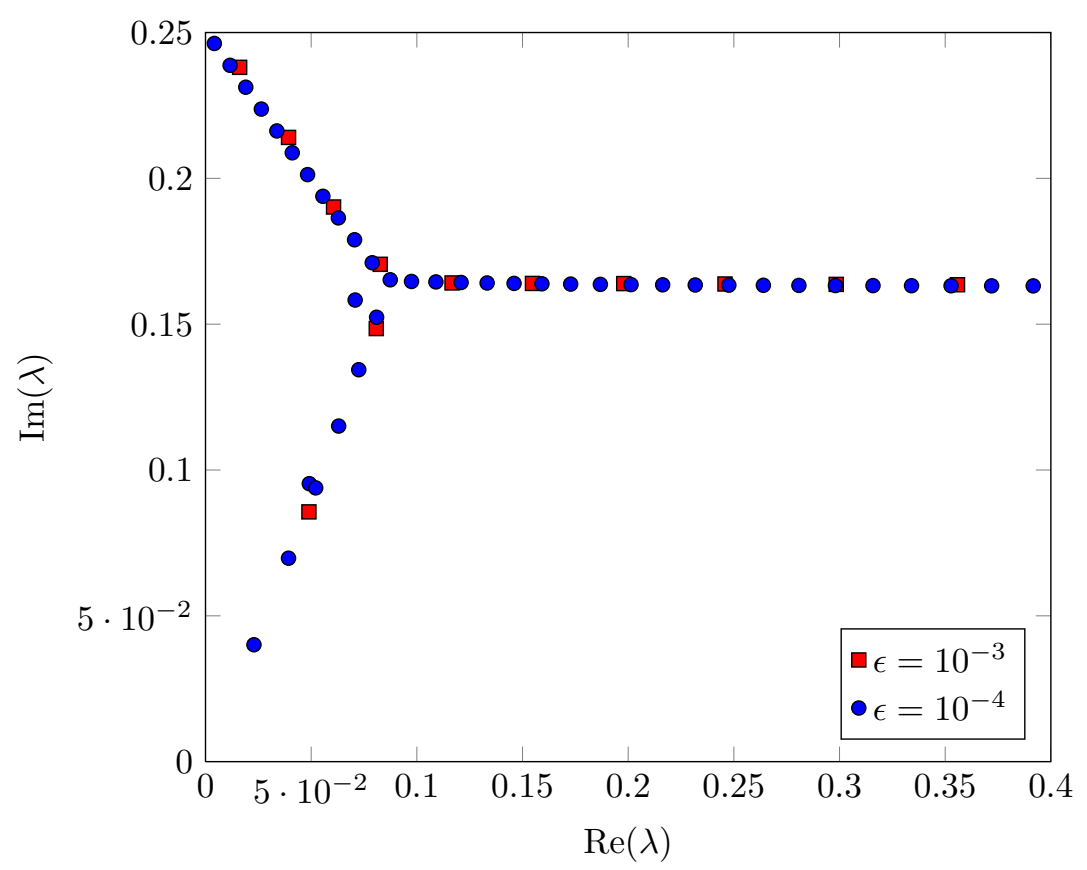

Figure 3: Spectral graph for the Poiseuille profile for two values of $\epsilon$.
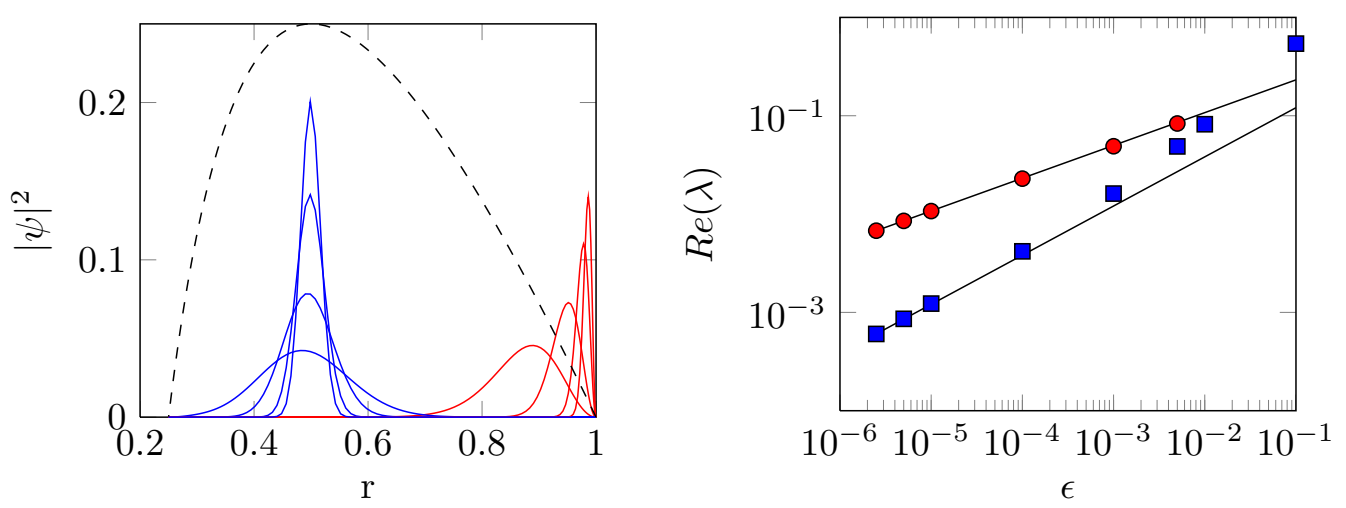

Figure 4: Left panel: Localization of eigenfunctions for the spectral branch emerging from the right end point (red) and the quadratic critical point (blue). Eigenfunctions shown for $\epsilon=\left[10 .^{-3}, 10^{-4}, 10^{-5}, 2.5 \times 10^{-6}\right]$ with $w(r)$ shown in black. The right panel shows scaling of each branch along with the prediction $\sim \epsilon^{1 / 3}$ and $\sim \epsilon^{1 / 2}$. 

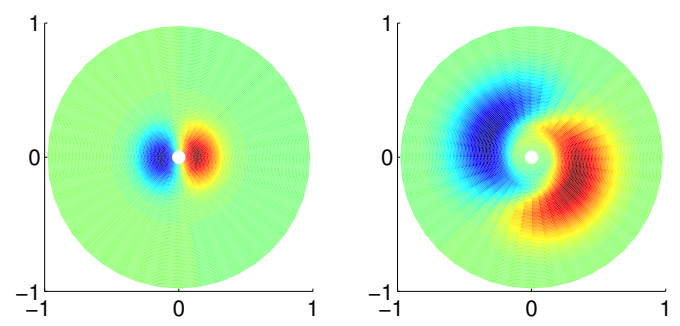

Figure 5: Evolution of the scalar tracer field in a time-dependent vortical velocity field. The figure on the left represents the initial condition, the figure on the right shows the state of the system after 10 periods.

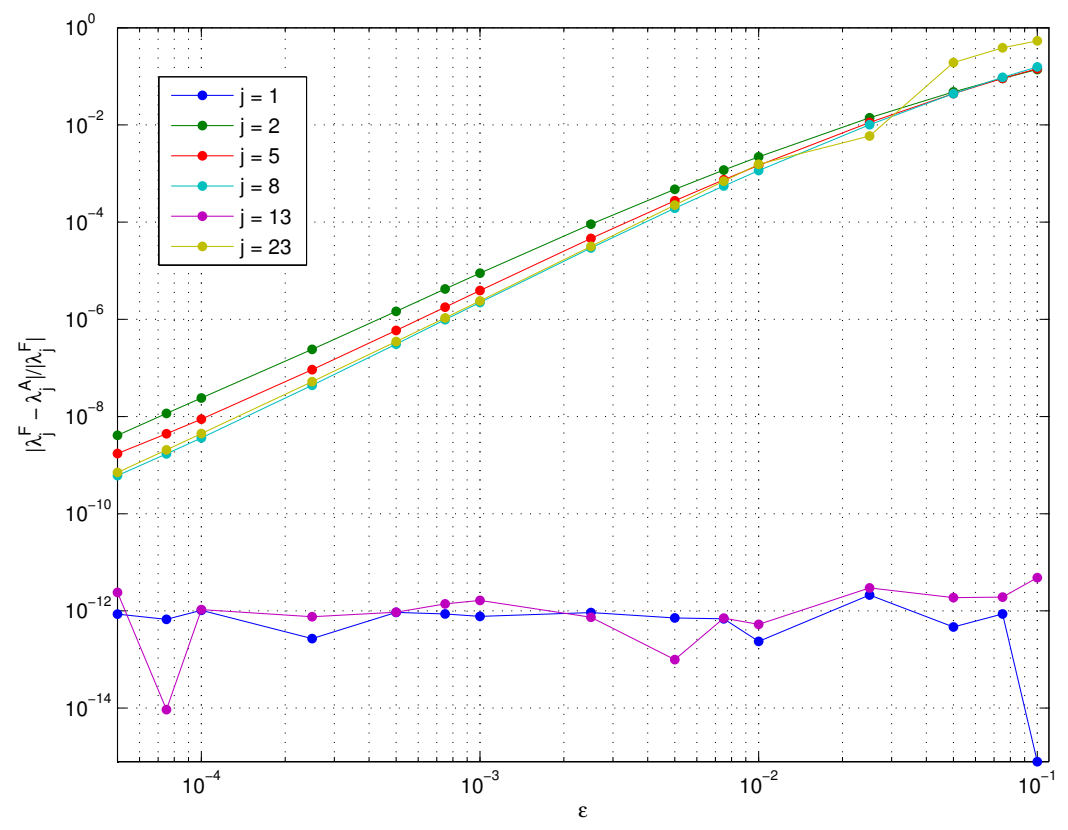

Figure 6: Difference in the calculation of eigenvalues of the full and average operators (as given by (57)) as a function of the parameter $\varepsilon$. 


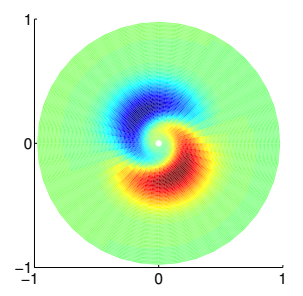

(a) $t=T$

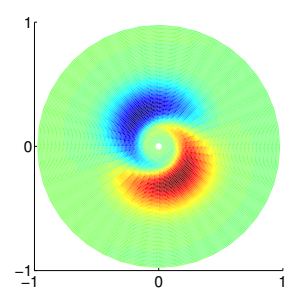

(e) $t=T$

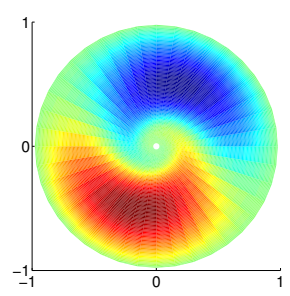

(b) $t=10 T$

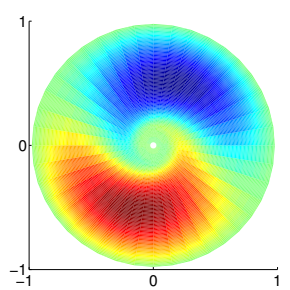

(f) $t=10 T$

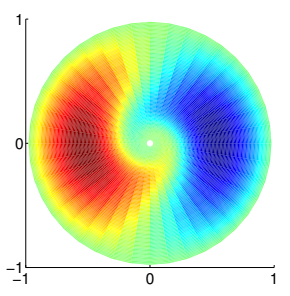

(c) $t=20 T$

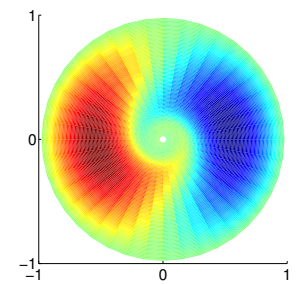

(g) $t=20 T$

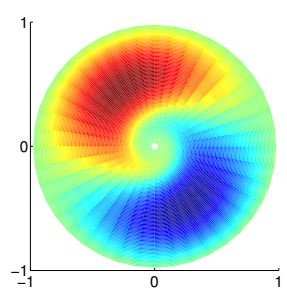

(d) $t=30 T$

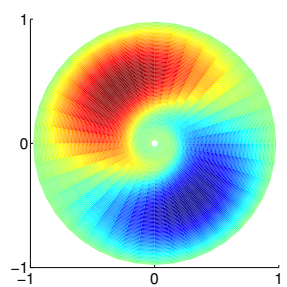

(h) $t=30 T$

Figure 7: Full time-dependent and averaged dynamics of the tracers under the influence of the constant mean flow shown for 1, 10, 20, and 30 periods. The top row shows results for the full operator and bottom row for the averaged evolution.

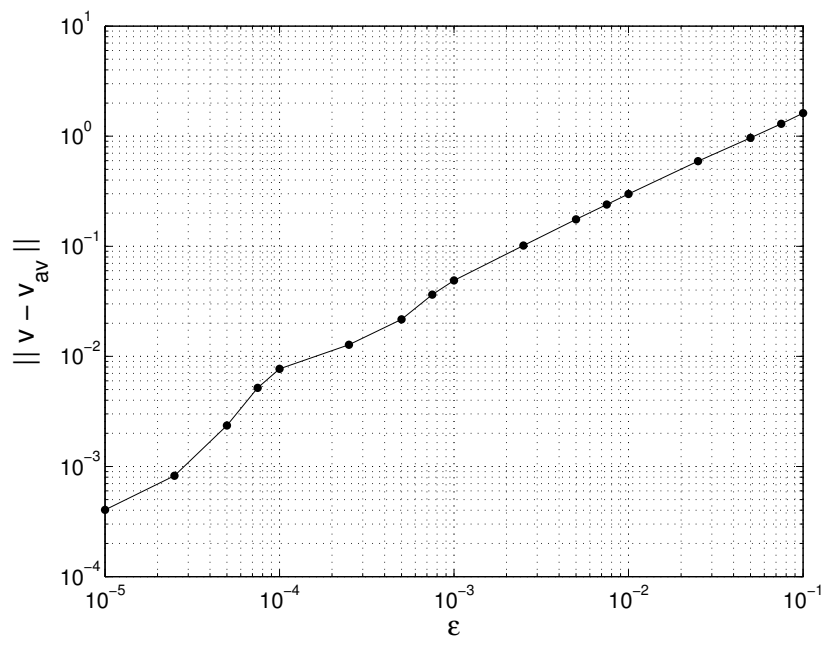

Figure 8: $L^{2}$-norm of a difference between solutions to full and approximate equations as a function of parameter $\varepsilon$. Convergence rate is found to be $\sim \varepsilon^{0.88}$. 


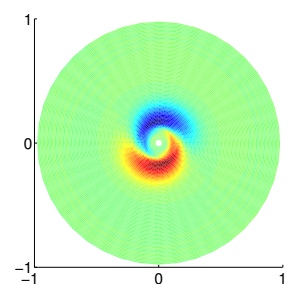

(a) $t=20 T$

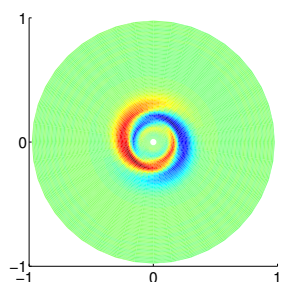

(b) $t=100 T$

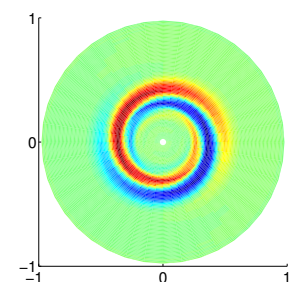

(c) $t=500 T$

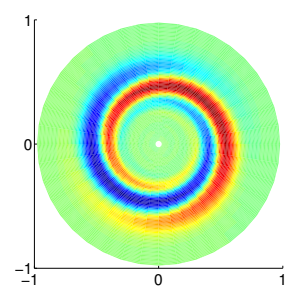

(d) $t=1000 T$

Figure 9: Large-time evolution if the tracer field for the case of extremely small diffusivity $\varepsilon=10^{-5}$. 